\title{
Cross sections for 2-to-3 meson-meson scattering
}

\author{
Wan-Xia Li, ${ }^{1}$ Xiao-Ming Xu๑, ${ }^{1}$ and H. J. Weber ${ }^{2}$ \\ ${ }^{1}$ Department of Physics, Shanghai University, Baoshan, Shanghai 200444, China \\ ${ }^{2}$ Department of Physics, University of Virginia, Charlottesville, Virginia 22904, USA
}

(Received 27 June 2019; published 28 January 2020)

\begin{abstract}
We study 2-to-3 meson-meson scattering based on the process that a gluon is created from a constituent quark or antiquark and subsequently the gluon creates a quark-antiquark pair. The transition potential for the process is derived in QCD. Eight Feynman diagrams at tree level are involved in the 2-to-3 mesonmeson scattering. Starting from the $S$-matrix element, we derive the unpolarized cross section from the eight transition amplitudes corresponding to the eight Feynman diagrams. The transition amplitudes contain color, spin, and flavor matrix elements. The 2-to-3 meson-meson scattering includes $\pi \pi \rightarrow \pi K \bar{K}$, $\pi K \rightarrow \pi \pi K, \pi K \rightarrow K K \bar{K}, K K \rightarrow \pi K K$, and $K \bar{K} \rightarrow \pi K \bar{K}$. Cross sections for the reactions are calculated. The cross sections depend on temperature obviously, and the cross section for $\pi K \rightarrow \pi \pi K$ for total isospin $I=3 / 2$ at zero temperature is compared to experimental data. By comparison with inelastic 2-to-2 mesonmeson scattering, we find that 2-to-3 meson-meson scattering may be as important as inelastic 2-to-2 meson-meson scattering.
\end{abstract}

DOI: 10.1103/PhysRevD.101.014025

\section{INTRODUCTION}

Many experiments and analyses have been done for elastic $\pi \pi$ scattering, elastic $\pi K$ scattering, and elastic $K \bar{K}$ scattering. Elastic phase shifts and elastic cross sections for $\pi \pi$ scattering have been measured via $\pi^{-} p \rightarrow \pi^{-} \pi^{-} \Delta^{++}$ [1-3], $\pi^{+} p \rightarrow \pi^{0} \pi^{0} \Delta^{++}$[4], $\pi^{+} p \rightarrow \pi^{+} \pi^{+} n$ [5-7], $\pi^{-} p \rightarrow$ $\pi^{0} \pi^{0} n[8], \pi^{-} d \rightarrow \pi^{-} \pi^{-} p p[9,10], \pi^{+} p \rightarrow \pi^{+} \pi^{-} \Delta^{++}[11]$, $\pi^{ \pm} p \rightarrow \pi^{ \pm} \pi^{0} p[7,12,13], \pi^{-} p \rightarrow \pi^{-} \pi^{+} n$ [7,14-21], and $K^{ \pm} \rightarrow \pi^{+} \pi^{-} e^{ \pm} \nu[22-25]$. When the total isospin of the two pions is 0 or 1 , from phase shifts and cross sections one can identify resonances such as $f_{0}(980), f_{2}(1270), f_{0}(1370)$, $a_{0}(980)$, and $\rho(1450)$. When the total isospin equals 2 , the cross section for elastic $\pi \pi$ scattering can go up to $12 \mathrm{mb}$. Elastic phase shifts and elastic cross sections for $\pi K$ scattering have been measured via $K^{ \pm} p \rightarrow K^{ \pm} \pi^{-} \Delta^{++}$ [26-29], $K^{+} p \rightarrow K^{0} \pi^{0} \Delta^{++}$[26], $K^{ \pm} p \rightarrow K^{ \pm} \pi^{+} n$ [29,30], and $D^{+} \rightarrow K^{-} \pi^{+} e^{+} \nu_{e}$ [31]. From elastic $\pi K$ scattering with a total isospin of $1 / 2$, one can find resonances such as $K_{0}^{*}(1430)$. The cross section for elastic $\pi K$ scattering with the other total isospin $3 / 2$ may become as large as $4.6 \mathrm{mb}$. The elastic cross section for $K^{+} K^{-}$scattering obtained from $\pi^{+} p \rightarrow K^{+} K^{-} \Delta^{++}$in Ref. [11] decreases from $3.8 \mathrm{mb}$ at $1 \mathrm{GeV}$ to $2.9 \mathrm{mb}$ at $1.38 \mathrm{GeV}$. Elastic phase

Published by the American Physical Society under the terms of the Creative Commons Attribution 4.0 International license. Further distribution of this work must maintain attribution to the author(s) and the published article's title, journal citation, and DOI. Funded by SCOAP ${ }^{3}$. shifts for $K^{+} K^{-}$scattering were extracted from $\pi^{-} p \rightarrow$ $K^{+} K^{-} n$ and $\pi^{+} n \rightarrow K^{+} K^{-} p$ in Ref. [32]. A variety of theoretical approaches [33-46] apply to elastic mesonmeson scattering.

Experimental data on $\pi \pi \rightarrow \rho$ are given in Ref. [47] and the cross section for $\pi K \rightarrow K^{*}$ in vacuum was estimated in Ref. [48]. On the basis of the process that a quark in one initial meson and an antiquark in the other initial meson annihilate into a gluon and subsequently the gluon is absorbed by the other antiquark or quark, 2-to-1 mesonmeson scattering has been studied in Ref. [49], and the resulting cross sections for $\pi \pi \rightarrow \rho$ and $\pi K \rightarrow K^{*}$ agree with the empirical data given in Refs. [47,48]. Cross sections for the reactions $\pi^{-} \pi^{-} \rightarrow \pi^{-} \pi^{-} \pi^{+} \pi^{-}$and $\pi^{-} \pi^{-} \rightarrow$ $\pi^{-} \pi^{-} \pi^{0} \pi^{0}$ were measured in Refs. [3,9], and the cross section goes up when the dipion mass increases from $0.8 \mathrm{GeV}$. The cross section for $\pi^{-} K^{-} \rightarrow \pi^{-} \pi^{-} K^{0}+$ $\pi^{0} \pi^{-} K^{-}$was investigated in Ref. [27]. In the present work we study 2-to-3 meson-meson scattering based on the process that a gluon is created from a quark or an antiquark in the two initial mesons, and the gluon then creates a quark and an antiquark. We note that 2-to-3 meson-meson scattering has not yet been studied in theory.

Inelastic 2-to-2 meson-meson scattering has been studied in Refs. [50-54]. The reactions $\pi \pi \rightarrow K \bar{K}, \rho \rho \rightarrow K \bar{K}$, $\pi \rho \rightarrow K \bar{K}^{*}$, and $\pi \rho \rightarrow K^{*} \bar{K}$ can be described by effective meson Lagrangians. The cross sections for the four reactions have been obtained from the exchange of either a kaon or a vector kaon between the two colliding mesons [50,51]. A study of $\pi \pi \rightarrow K \bar{K}$ scattering by means of partial-wave dispersion relations of the Roy-Steiner type is 
performed in Ref. [54], and precise parametrizations of the $S, P$, and $D$ partial waves in the $\pi \pi \rightarrow K \bar{K}$ scattering amplitude are obtained from the data. To generate all the resonances with isospin 0 and masses below $2 \mathrm{GeV}$, $S$-wave meson-meson scattering for total isospin $I=0$ and $1 / 2$ is studied in Ref. [55] with 13 coupled channels. Their $S$-wave phase shifts and modulus for $\pi \pi \rightarrow K \bar{K}$ for $I=0$ agree with experimental data, and $\pi \pi \rightarrow \rho \rho$ is determined by minimal coupling. In terms of quark degrees of freedom some reactions are mainly governed by quark interchange, quark-antiquark annihilation and creation, or both. For example, $\pi \pi \rightarrow \rho \rho$ for total isospin $I=2$ and $\pi K \rightarrow \rho K^{*}$ for $I=3 / 2$ involve quark interchange [52]; $\pi \pi \rightarrow \rho \rho$ for $I=1$ and $\pi \rho \rightarrow K \bar{K}^{*}$ involve quark-antiquark annihilation and creation [53]; $\pi \pi \rightarrow \rho \rho$ for $I=0$ and $\pi K \rightarrow \rho K^{*}$ for $I=1 / 2$ involve quark interchange as well as quark-antiquark annihilation and creation $[49,53]$. The reactions governed by quark interchange as well as quarkantiquark annihilation and creation have the characteristic feature that close to threshold quark interchange dominates the reactions near the critical temperature, and in the other energy region quark-antiquark annihilation and creation may dominate the reactions.

In hadronic matter created in relativistic heavy-ion collisions at the Relativistic Heavy Ion Collider and at
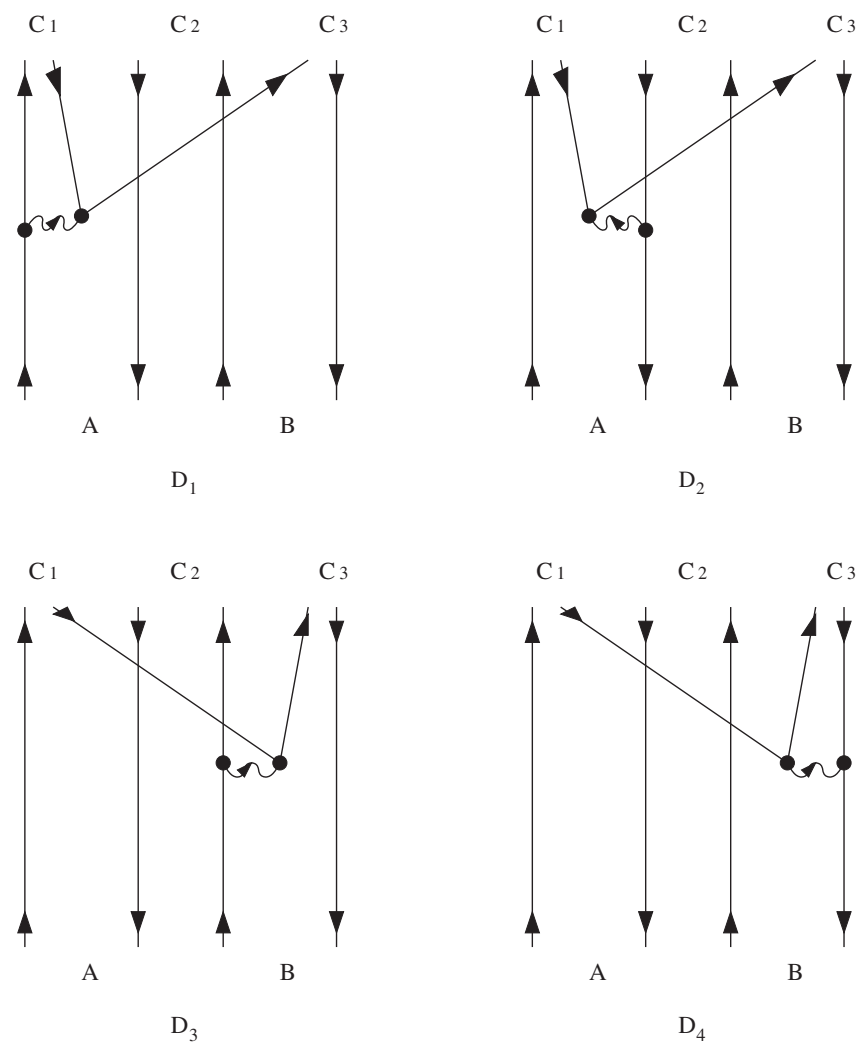

FIG. 1. Reaction $A\left(q_{1} \bar{q}_{1}\right)+B\left(q_{2} \bar{q}_{2}\right) \rightarrow C_{1}\left(q_{1} \bar{q}_{4}\right)+C_{2}\left(q_{2} \bar{q}_{1}\right)+$ $C_{3}\left(q_{3} \bar{q}_{2}\right)$. Solid lines with up (down) arrows represent quarks (antiquarks). Wavy lines represent gluons. the Large Hadron Collider, thermal equilibrium is established by elastic meson-meson scattering. Since inelastic meson-meson scattering alters the meson number, chemical equilibrium is determined by inelastic meson-meson scattering. Exactly how thermal equilibrium is established and how chemical equilibrium is established are two important issues of hadronic matter. In lead-lead collisions and in xenon-xenon collisions at the Large Hadron Collider, meson momentum measured by the ATLAS Collaboration, the CMS Collaboration, and the ALICE Collaboration goes up to $1000 \mathrm{GeV} / c$ [56-58]. A meson of such large momenta in collision with another meson in hadronic matter may yield three or more mesons. Two-to-three meson-meson scattering affects chemical equilibrium. Therefore, we need to study the 2-to-3 meson-meson scattering in hadronic matter.

This paper is organized as follows. In Sec. II we present eight Feynman diagrams for 2-to-3 meson-meson scattering, the transition amplitudes corresponding to the eight diagrams, and cross sections related to the transition amplitudes. In Sec. III we derive a transition potential for the process that a gluon is created from a quark or an antiquark and the gluon creates a quark and an antiquark. In Sec. IV we calculate color, spin, and flavor matrix elements in the transition amplitudes. In Sec. V numerical cross
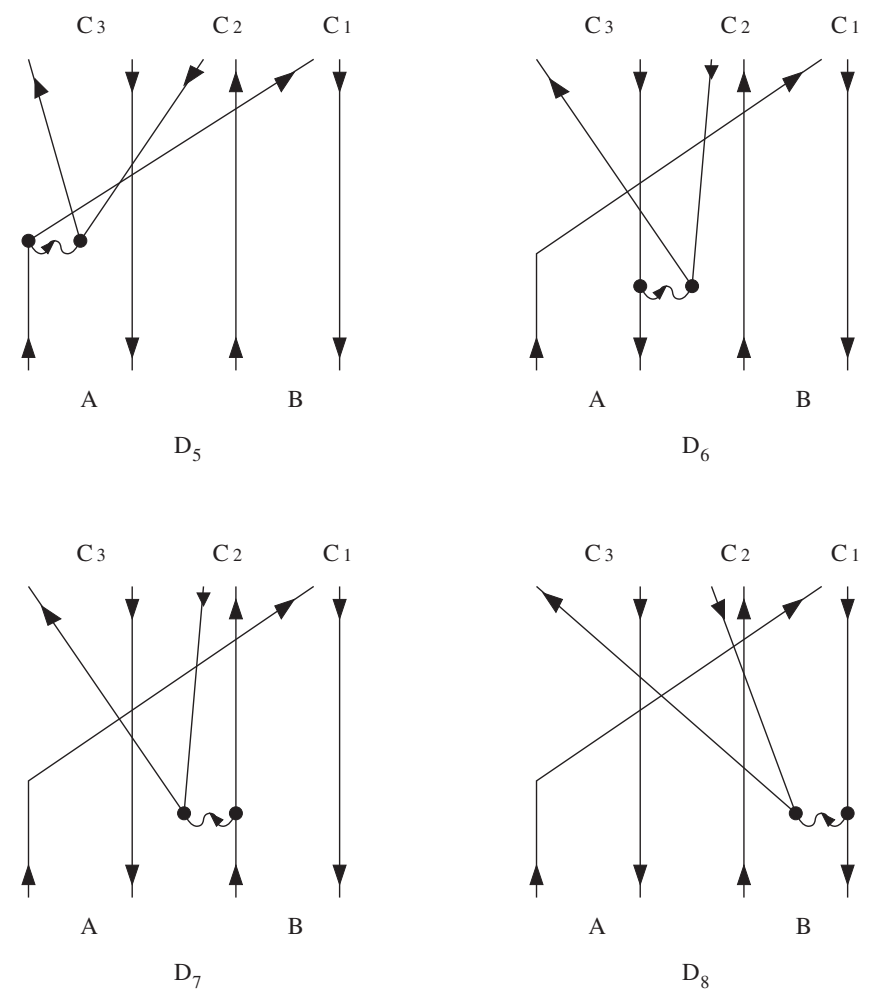

FIG. 2. Reaction $A\left(q_{1} \bar{q}_{1}\right)+B\left(q_{2} \bar{q}_{2}\right) \rightarrow C_{1}\left(q_{1} \bar{q}_{2}\right)+C_{2}\left(q_{2} \bar{q}_{4}\right)+$ $C_{3}\left(q_{3} \bar{q}_{1}\right)$. Solid lines with up (down) arrows represent quarks (antiquarks). Wavy lines represent gluons. 
sections are presented and relevant discussions are given. In Sec. VI we summarize the present work.

\section{CROSS-SECTION FORMULAS}

Meson $A$ contains quark $q_{1}$ and antiquark $\bar{q}_{1}$, and meson $B$ has quark $q_{2}$ and antiquark $\bar{q}_{2}$. In the collision of mesons $A$ and $B$ a constituent quark or antiquark may emit a virtual gluon which subsequently splits into quark $q_{3}$ and antiquark $\bar{q}_{4}$. The three quarks and antiquarks then combine into mesons $C_{1}, C_{2}$, and $C_{3}$. Four Feynman diagrams are shown in Fig. 1 for $A\left(q_{1} \bar{q}_{1}\right)+B\left(q_{2} \bar{q}_{2}\right) \rightarrow$
$C_{1}\left(q_{1} \bar{q}_{4}\right)+C_{2}\left(q_{2} \bar{q}_{1}\right)+C_{3}\left(q_{3} \bar{q}_{2}\right)$, and four other diagrams in Fig. 2 for $A\left(q_{1} \bar{q}_{1}\right)+B\left(q_{2} \bar{q}_{2}\right) \rightarrow C_{1}\left(q_{1} \bar{q}_{2}\right)+$ $C_{2}\left(q_{2} \bar{q}_{4}\right)+C_{3}\left(q_{3} \bar{q}_{1}\right)$. Diagram $\mathrm{D}_{1}\left(\mathrm{D}_{2}, \mathrm{D}_{3}, \mathrm{D}_{4}\right)$ in Fig. 1 involves the emission of a gluon from $q_{1}\left(\bar{q}_{1}, q_{2}\right.$, $\bar{q}_{2}$ ) and the subsequent splitting of the gluon into $q_{3}$ and $\bar{q}_{4}$, and diagram $\mathrm{D}_{5}\left(\mathrm{D}_{6}, \mathrm{D}_{7}, \mathrm{D}_{8}\right)$ in Fig. 2 also involves this process. Denote the energy of meson $A\left(B, C_{1}, C_{2}, C_{3}\right)$ by $E_{A}\left(E_{B}, E_{C_{1}}, E_{C_{2}}, E_{C_{3}}\right)$. The total energy of the two initial mesons is $E_{\mathrm{i}}=E_{A}+E_{B}$, and the total energy of the three final mesons is $E_{\mathrm{f}}=E_{C_{1}}+E_{C_{2}}+E_{C_{3}}$. The $S$-matrix element for $A+B \rightarrow C_{1}+C_{2}+C_{3}$ is

$$
\begin{aligned}
S_{\mathrm{fi}}= & \delta_{\mathrm{fi}}-2 \pi i \delta\left(E_{\mathrm{f}}-E_{\mathrm{i}}\right)\left(\left\langle C_{1}, C_{2}, C_{3}\left|V_{\mathrm{D}_{1}}\right| A, B\right\rangle+\left\langle C_{1}, C_{2}, C_{3}\left|V_{\mathrm{D}_{2}}\right| A, B\right\rangle+\left\langle C_{1}, C_{2}, C_{3}\left|V_{\mathrm{D}_{3}}\right| A, B\right\rangle+\left\langle C_{1}, C_{2}, C_{3}\left|V_{\mathrm{D}_{4}}\right| A, B\right\rangle\right. \\
& \left.+\left\langle C_{1}, C_{2}, C_{3}\left|V_{\mathrm{D}_{5}}\right| A, B\right\rangle+\left\langle C_{1}, C_{2}, C_{3}\left|V_{\mathrm{D}_{6}}\right| A, B\right\rangle+\left\langle C_{1}, C_{2}, C_{3}\left|V_{\mathrm{D}_{7}}\right| A, B\right\rangle+\left\langle C_{1}, C_{2}, C_{3}\left|V_{\mathrm{D}_{8}}\right| A, B\right\rangle\right),
\end{aligned}
$$

where $V_{\mathrm{D}_{1}}\left(V_{\mathrm{D}_{2}}, V_{\mathrm{D}_{3}}, V_{\mathrm{D}_{4}}\right)$ represents the transition potential for $q_{1} \rightarrow q_{1}+q_{3}+\bar{q}_{4} \quad\left(\bar{q}_{1} \rightarrow \bar{q}_{1}+q_{3}+\bar{q}_{4}\right.$, $\left.q_{2} \rightarrow q_{2}+q_{3}+\bar{q}_{4}, \quad \bar{q}_{2} \rightarrow \bar{q}_{2}+q_{3}+\bar{q}_{4}\right)$ in diagram $\mathrm{D}_{1}$ $\left(\mathrm{D}_{2}, \mathrm{D}_{3}, \mathrm{D}_{4}\right)$, and $V_{\mathrm{D}_{5}}\left(V_{\mathrm{D}_{6}}, V_{\mathrm{D}_{7}}, V_{\mathrm{D}_{8}}\right)$ represents the transition potential for $q_{1} \rightarrow q_{1}+q_{3}+\bar{q}_{4}\left(\bar{q}_{1} \rightarrow \bar{q}_{1}+q_{3}+\bar{q}_{4}\right.$, $\left.q_{2} \rightarrow q_{2}+q_{3}+\bar{q}_{4}, \quad \bar{q}_{2} \rightarrow \bar{q}_{2}+q_{3}+\bar{q}_{4}\right)$ in diagram $\mathrm{D}_{5}$ $\left(\mathrm{D}_{6}, \mathrm{D}_{7}, \mathrm{D}_{8}\right)$. Let $\vec{P}_{a b}, \vec{R}_{a b}$, and $\vec{r}_{a b}$ be the total momentum, the center-of-mass coordinate, and the relative coordinate of constituents $a$ and $b$, respectively. The wave function $|A, B\rangle$ of mesons $A$ and $B$ is

$\psi_{q_{1} \bar{q}_{1}, q_{2} \bar{q}_{2}}=\frac{e^{i \vec{P}_{q_{1}} \bar{q}_{1} \cdot \vec{R}_{q_{1} \bar{q}_{1}}}}{\sqrt{V}} \psi_{q_{1} \bar{q}_{1}}\left(\vec{r}_{q_{1} \bar{q}_{1}}\right) \frac{e^{i \vec{P}_{q_{2} \bar{q}_{2}} \cdot \vec{R}_{q_{2}} \bar{q}_{2}}}{\sqrt{V}} \psi_{q_{2} \bar{q}_{2}}\left(\vec{r}_{q_{2} \bar{q}_{2}}\right)$.

The wave function $\left|C_{1}, C_{2}, C_{3}\right\rangle$ of mesons $C_{1}, C_{2}$, and $C_{3}$ is

$$
\begin{aligned}
\psi_{q_{1} \bar{q}_{4}, q_{2} \bar{q}_{1}, q_{3} \bar{q}_{2}}= & \frac{e^{i \vec{P}_{q_{1}} \cdot \vec{q}_{4} \cdot \vec{q}_{q_{1}} \bar{q}_{4}}}{\sqrt{V}} \psi_{q_{1} \bar{q}_{4}}\left(\vec{r}_{q_{1} \bar{q}_{4}}\right) \\
& \times \frac{e^{i \vec{P}_{q_{2} \bar{q}_{1}} \cdot \vec{R}_{q_{2} \bar{q}_{1}}}}{\sqrt{V}} \psi_{q_{2} \bar{q}_{1}}\left(\vec{r}_{q_{2} \bar{q}_{1}}\right) \\
& \times \frac{e^{i \vec{P}_{q_{3} \bar{q}_{2}} \cdot \vec{R}_{q_{3} \bar{q}_{2}}}}{\sqrt{V}} \psi_{q_{3} \bar{q}_{2}}\left(\vec{r}_{q_{3} \bar{q}_{2}}\right),
\end{aligned}
$$

corresponding to the four diagrams in Fig. 1 or

$$
\begin{aligned}
\psi_{q_{1} \bar{q}_{2}, q_{2} \bar{q}_{4}, q_{3} \bar{q}_{1}}= & \frac{e^{i \vec{P}_{q_{1}} \bar{q}_{2} \cdot \vec{R}_{q_{1}} \bar{q}_{2}}}{\sqrt{V}} \psi_{q_{1} \bar{q}_{2}}\left(\vec{r}_{q_{1} \bar{q}_{2}}\right) \\
& \times \frac{e^{i \vec{P}_{q_{2}} \bar{q}_{4} \cdot \vec{R}_{q_{2}} \bar{q}_{4}}}{\sqrt{V}} \psi_{q_{2} \bar{q}_{4}}\left(\vec{r}_{q_{2} \bar{q}_{4}}\right) \\
& \times \frac{e^{i \vec{P}_{q_{3}} \bar{q}_{1} \cdot \vec{R}_{q_{3}} \bar{q}_{1}}}{\sqrt{V}} \psi_{q_{3} \bar{q}_{1}}\left(\vec{r}_{q_{3} \bar{q}_{1}}\right),
\end{aligned}
$$

corresponding to the four diagrams in Fig. 2. The mesonic quark-antiquark wave function $\psi_{a b}\left(\vec{r}_{a b}\right)$ is the product of the color wave function, the spin wave function, the flavor wave function, and the relative-motion wave function of constituents $a$ and $b$. Every meson wave function is normalized in the volume $V$.

From the $S$-matrix element we derive the transition amplitudes corresponding to the eight Feynman diagrams in Figs. 1 and 2. From the transition amplitudes we obtain the unpolarized cross section for $A+B \rightarrow C_{1}+C_{2}+C_{3}$. The position vector and the mass of constituent $c$ are denoted by $\vec{r}_{c}$ and $m_{c}$, respectively.

We first consider the four diagrams in Fig. 1. The five independent constituent position-vectors are $\vec{r}_{q_{1}}, \vec{r}_{\bar{q}_{1}}, \vec{r}_{q_{2}}$, $\vec{r}_{\bar{q}_{2}}$, and $\vec{r}_{q_{3}}$. They are related to the relative coordinates $\left(\vec{r}_{q_{1} \bar{q}_{1}}, \vec{r}_{q_{2} \bar{q}_{2}}\right)$ and the center-of-mass coordinates $\left(\vec{R}_{q_{1} \bar{q}_{4}}\right.$, $\left.\vec{R}_{q_{2} \bar{q}_{1}}, \vec{R}_{q_{3} \bar{q}_{2}}\right)$ by

$$
\begin{aligned}
\vec{r}_{q_{1}}= & \frac{m_{\bar{q}_{1}} m_{\bar{q}_{2}} m_{\bar{q}_{4}}}{m_{q_{1}} m_{q_{2}} m_{q_{3}}+m_{\bar{q}_{1}} m_{\bar{q}_{2}} m_{\bar{q}_{4}}} \vec{r}_{q_{1} \bar{q}_{1}}-\frac{m_{q_{2}} m_{\bar{q}_{2}} m_{\bar{q}_{4}}}{m_{q_{1}} m_{q_{2}} m_{q_{3}}+m_{\bar{q}_{1}} m_{\bar{q}_{2}} m_{\bar{q}_{4}}} \vec{r}_{q_{2} \bar{q}_{2}}+\frac{m_{q_{2}} m_{q_{3}}\left(m_{q_{1}}+m_{\bar{q}_{4}}\right)}{m_{q_{1}} m_{q_{2}} m_{q_{3}}+m_{\bar{q}_{1}} m_{\bar{q}_{2}} m_{\bar{q}_{4}}} \vec{R}_{q_{1} \bar{q}_{4}} \\
& +\frac{m_{\bar{q}_{2}} m_{\bar{q}_{4}}\left(m_{q_{2}}+m_{\bar{q}_{1}}\right)}{m_{q_{1}} m_{q_{2}} m_{q_{3}}+m_{\bar{q}_{1}} m_{\bar{q}_{2}} m_{\bar{q}_{4}}} \vec{R}_{q_{2} \bar{q}_{1}}-\frac{m_{q_{2}} m_{\bar{q}_{4}}\left(m_{q_{3}}+m_{\bar{q}_{2}}\right)}{m_{q_{1}} m_{q_{2}} m_{q_{3}}+m_{\bar{q}_{1}} m_{\bar{q}_{2}} m_{\bar{q}_{4}}} \vec{R}_{q_{3} \bar{q}_{2}},
\end{aligned}
$$




$$
\begin{aligned}
& \vec{r}_{\bar{q}_{1}}=-\frac{m_{q_{1}} m_{q_{2}} m_{q_{3}}}{m_{q_{1}} m_{q_{2}} m_{q_{3}}+m_{\bar{q}_{1}} m_{\bar{q}_{2}} m_{\bar{q}_{4}}} \vec{r}_{q_{1} \bar{q}_{1}}-\frac{m_{q_{2}} m_{\bar{q}_{2}} m_{\bar{q}_{4}}}{m_{q_{1}} m_{q_{2}} m_{q_{3}}+m_{\bar{q}_{1}} m_{\bar{q}_{2}} m_{\bar{q}_{4}}} \vec{r}_{q_{2} \bar{q}_{2}}+\frac{m_{q_{2}} m_{q_{3}}\left(m_{q_{1}}+m_{\bar{q}_{4}}\right)}{m_{q_{1}} m_{q_{2}} m_{q_{3}}+m_{\bar{q}_{1}} m_{\bar{q}_{2}} m_{\bar{q}_{4}}} \vec{R}_{q_{1} \bar{q}_{4}} \\
& +\frac{m_{\bar{q}_{2}} m_{\bar{q}_{4}}\left(m_{q_{2}}+m_{\bar{q}_{1}}\right)}{m_{q_{1}} m_{q_{2}} m_{q_{3}}+m_{\bar{q}_{1}} m_{\bar{q}_{2}} m_{\bar{q}_{4}}} \vec{R}_{q_{2} \bar{q}_{1}}-\frac{m_{q_{2}} m_{\bar{q}_{4}}\left(m_{q_{3}}+m_{\bar{q}_{2}}\right)}{m_{q_{1}} m_{q_{2}} m_{q_{3}}+m_{\bar{q}_{1}} m_{\bar{q}_{2}} m_{\bar{q}_{4}}} \vec{R}_{q_{3} \bar{q}_{2}}, \\
& \vec{r}_{q_{2}}=\frac{m_{q_{1}} m_{q_{3}} m_{\bar{q}_{1}}}{m_{q_{1}} m_{q_{2}} m_{q_{3}}+m_{\bar{q}_{1}} m_{\bar{q}_{2}} m_{\bar{q}_{4}}} \vec{r}_{q_{1} \bar{q}_{1}}+\frac{m_{\bar{q}_{1}} m_{\bar{q}_{2}} m_{\bar{q}_{4}}}{m_{q_{1}} m_{q_{2}} m_{q_{3}}+m_{\bar{q}_{1}} m_{\bar{q}_{2}} m_{\bar{q}_{4}}} \vec{r}_{q_{2} \bar{q}_{2}}-\frac{m_{q_{3}} m_{\bar{q}_{1}}\left(m_{q_{1}}+m_{\bar{q}_{4}}\right)}{m_{q_{1}} m_{q_{2}} m_{q_{3}}+m_{\bar{q}_{1}} m_{\bar{q}_{2}} m_{\bar{q}_{4}}} \vec{R}_{q_{1} \bar{q}_{4}} \\
& +\frac{m_{q_{1}} m_{q_{3}}\left(m_{q_{2}}+m_{\bar{q}_{1}}\right)}{m_{q_{1}} m_{q_{2}} m_{q_{3}}+m_{\bar{q}_{1}} m_{\bar{q}_{2}} m_{\bar{q}_{4}}} \vec{R}_{q_{2} \bar{q}_{1}}+\frac{m_{\bar{q}_{1}} m_{\bar{q}_{4}}\left(m_{q_{3}}+m_{\bar{q}_{2}}\right)}{m_{q_{1}} m_{q_{2}} m_{q_{3}}+m_{\bar{q}_{1}} m_{\bar{q}_{2}} m_{\bar{q}_{4}}} \vec{R}_{q_{3} \bar{q}_{2}}, \\
& \vec{r}_{\bar{q}_{2}}=\frac{m_{q_{1}} m_{q_{3}} m_{\bar{q}_{1}}}{m_{q_{1}} m_{q_{2}} m_{q_{3}}+m_{\bar{q}_{1}} m_{\bar{q}_{2}} m_{\bar{q}_{4}}} \vec{r}_{q_{1} \bar{q}_{1}}-\frac{m_{q_{1}} m_{q_{2}} m_{q_{3}}}{m_{q_{1}} m_{q_{2}} m_{q_{3}}+m_{\bar{q}_{1}} m_{\bar{q}_{2}} m_{\bar{q}_{4}}} \vec{r}_{q_{2} \bar{q}_{2}}-\frac{m_{q_{3}} m_{\bar{q}_{1}}\left(m_{q_{1}}+m_{\bar{q}_{4}}\right)}{m_{q_{1}} m_{q_{2}} m_{q_{3}}+m_{\bar{q}_{1}} m_{\bar{q}_{2}} m_{\bar{q}_{4}}} \vec{R}_{q_{1} \bar{q}_{4}} \\
& +\frac{m_{q_{1}} m_{q_{3}}\left(m_{q_{2}}+m_{\bar{q}_{1}}\right)}{m_{q_{1}} m_{q_{2}} m_{q_{3}}+m_{\bar{q}_{1}} m_{\bar{q}_{2}} m_{\bar{q}_{4}}} \vec{R}_{q_{2} \bar{q}_{1}}+\frac{m_{\bar{q}_{1}} m_{\bar{q}_{4}}\left(m_{q_{3}}+m_{\bar{q}_{2}}\right)}{m_{q_{1}} m_{q_{2}} m_{q_{3}}+m_{\bar{q}_{1}} m_{\bar{q}_{2}} m_{\bar{q}_{4}}} \vec{R}_{q_{3} \bar{q}_{2}}, \\
& \vec{r}_{q_{3}}=-\frac{m_{q_{1}} m_{\bar{q}_{1}} m_{\bar{q}_{2}}}{m_{q_{1}} m_{q_{2}} m_{q_{3}}+m_{\bar{q}_{1}} m_{\bar{q}_{2}} m_{\bar{q}_{4}}} \vec{r}_{q_{1} \bar{q}_{1}}+\frac{m_{q_{1}} m_{q_{2}} m_{\bar{q}_{2}}}{m_{q_{1}} m_{q_{2}} m_{q_{3}}+m_{\bar{q}_{1}} m_{\bar{q}_{2}} m_{\bar{q}_{4}}} \vec{r}_{q_{2} \bar{q}_{2}}+\frac{m_{\bar{q}_{1}} m_{\bar{q}_{2}}\left(m_{q_{1}}+m_{\bar{q}_{4}}\right)}{m_{q_{1}} m_{q_{2}} m_{q_{3}}+m_{\bar{q}_{1}} m_{\bar{q}_{2}} m_{\bar{q}_{4}}} \vec{R}_{q_{1} \bar{q}_{4}} \\
& -\frac{m_{q_{1}} m_{\bar{q}_{2}}\left(m_{q_{2}}+m_{\bar{q}_{1}}\right)}{m_{q_{1}} m_{q_{2}} m_{q_{3}}+m_{\bar{q}_{1}} m_{\bar{q}_{2}} m_{\bar{q}_{4}}} \vec{R}_{q_{2} \bar{q}_{1}}+\frac{m_{q_{1}} m_{q_{2}}\left(m_{q_{3}}+m_{\bar{q}_{2}}\right)}{m_{q_{1}} m_{q_{2}} m_{q_{3}}+m_{\bar{q}_{1}} m_{\bar{q}_{2}} m_{\bar{q}_{4}}} \vec{R}_{q_{3} \bar{q}_{2}},
\end{aligned}
$$

which lead to

$$
d \vec{r}_{q_{1}} d \vec{r}_{\bar{q}_{1}} d \vec{r}_{q_{2}} d \vec{r}_{\bar{q}_{2}} d \vec{r}_{q_{3}}=\frac{\left(m_{q_{1}}+m_{\bar{q}_{4}}\right)^{3}\left(m_{q_{2}}+m_{\bar{q}_{1}}\right)^{3}\left(m_{q_{3}}+m_{\bar{q}_{2}}\right)^{3}}{\left(m_{q_{1}} m_{q_{2}} m_{q_{3}}+m_{\bar{q}_{1}} m_{\bar{q}_{2}} m_{\bar{q}_{4}}\right)^{3}} d \vec{r}_{q_{1} \bar{q}_{1}} d \vec{r}_{q_{2} \bar{q}_{2}} d \vec{R}_{q_{1} \bar{q}_{4}} d \vec{R}_{q_{2} \bar{q}_{1}} d \vec{R}_{q_{3} \bar{q}_{2}} .
$$

Let $\vec{R}_{\text {total }}$ be the center-of-mass coordinate of the initial or final mesons. Denote the three-dimensional momentum of meson $A\left(B, C_{1}, C_{2}, C_{3}\right)$ by $\vec{P}_{A}\left(\vec{P}_{B}, \vec{P}_{C_{1}}, \vec{P}_{C_{2}}, \vec{P}_{C_{3}}\right)$. The total momentum of the two initial mesons is $\vec{P}_{\mathrm{i}}=\vec{P}_{A}+\vec{P}_{B}$, and the total momentum of the three final mesons is $\vec{P}_{\mathrm{f}}=\vec{P}_{C_{1}}+\vec{P}_{C_{2}}+\vec{P}_{C_{3}}$. From the transition potential $V_{\mathrm{D}_{1}}$ and the wave functions of the initial and final mesons, we have for diagram $\mathrm{D}_{1}$ :

$$
\begin{aligned}
& \left\langle C_{1}, C_{2}, C_{3}\left|V_{\mathrm{D}_{1}}\right| A, B\right\rangle=\left\langle q_{1} \bar{q}_{4}, q_{2} \bar{q}_{1}, q_{3} \bar{q}_{2}\left|V_{\mathrm{D}_{1}}\right| q_{1} \bar{q}_{1}, q_{2} \bar{q}_{2}\right\rangle \\
& =\int d \vec{r}_{q_{1}} d \vec{r}_{\bar{q}_{1}} d \vec{r}_{q_{2}} d \vec{r}_{\bar{q}_{2}} d \vec{r}_{q_{3}} \frac{e^{-i \vec{p}_{q_{1} \bar{q}_{4}} \cdot \vec{R}_{q_{1}} \bar{q}_{4}}}{\sqrt{V}} \psi_{q_{1} \bar{q}_{4}}^{+}\left(\vec{r}_{q_{1} \bar{q}_{4}}\right) \frac{e^{-i \vec{P}_{q_{2} \bar{q}_{1}} \cdot \vec{R}_{q_{2}} \bar{q}_{1}}}{\sqrt{V}} \psi_{q_{2} \bar{q}_{1}}^{+}\left(\vec{r}_{q_{2} \bar{q}_{1}}\right) \\
& \times \frac{e^{-i \vec{P}_{q_{3}} \bar{q}_{2} \cdot \vec{R}_{q_{3}} \bar{q}_{2}}}{\sqrt{V}} \psi_{q_{3} \bar{q}_{2}}^{+}\left(\vec{r}_{q_{3} \bar{q}_{2}}\right) V_{\mathrm{D}_{1}} \frac{e^{i \vec{P}_{q_{1} \bar{q}_{1}} \cdot \vec{R}_{q_{1} \bar{q}_{1}}}}{\sqrt{V}} \psi_{q_{1} \bar{q}_{1}}\left(\vec{r}_{q_{1} \bar{q}_{1}}\right) \frac{e^{i \vec{P}_{q_{2}} \bar{q}_{2} \cdot \vec{R}_{q_{2}} \bar{q}_{2}}}{\sqrt{V}} \psi_{q_{2} \bar{q}_{2}}\left(\vec{r}_{q_{2} \bar{q}_{2}}\right) \\
& =\frac{\left(m_{q_{1}}+m_{\bar{q}_{4}}\right)^{3}\left(m_{q_{2}}+m_{\bar{q}_{1}}\right)^{3}\left(m_{q_{3}}+m_{\bar{q}_{2}}\right)^{3}}{\sqrt{V^{5}}\left(m_{q_{1}} m_{q_{2}} m_{q_{3}}+m_{\bar{q}_{1}} m_{\bar{q}_{2}} m_{\bar{q}_{4}}\right)^{3}} \int d \vec{r}_{q_{1} \bar{q}_{1}} d \vec{r}_{q_{2} \bar{q}_{2}} d \vec{R}_{q_{1} \bar{q}_{4}} d \vec{R}_{q_{2} \bar{q}_{1}} d \vec{R}_{q_{3} \bar{q}_{2}} \\
& \times \psi_{q_{1} \bar{q}_{4}}^{+}\left(\vec{r}_{q_{1} \bar{q}_{4}}\right) \psi_{q_{2} \bar{q}_{1}}^{+}\left(\vec{r}_{q_{2} \bar{q}_{1}}\right) \psi_{q_{3} \bar{q}_{2}}^{+}\left(\vec{r}_{q_{3} \bar{q}_{2}}\right) V_{\mathrm{D}_{1}} \psi_{q_{1} \bar{q}_{1}}\left(\vec{r}_{q_{1} \bar{q}_{1}}\right) \psi_{q_{2} \bar{q}_{2}}\left(\vec{r}_{q_{2} \bar{q}_{2}}\right) \\
& \times \exp \left(-i \vec{P}_{q_{1} \bar{q}_{4}} \cdot \vec{R}_{q_{1} \bar{q}_{4}}-i \vec{P}_{q_{2} \bar{q}_{1}} \cdot \vec{R}_{q_{2} \bar{q}_{1}}-i \vec{P}_{q_{3} \bar{q}_{2}} \cdot \vec{R}_{q_{3} \bar{q}_{2}}+i \vec{P}_{q_{1} \bar{q}_{1}} \cdot \vec{R}_{q_{1} \bar{q}_{1}}+i \vec{P}_{q_{2} \bar{q}_{2}} \cdot \vec{R}_{q_{2} \bar{q}_{2}}\right) \text {, }
\end{aligned}
$$

where $\psi_{a b}^{+}$is the Hermitean conjugate of $\psi_{a b}$. In the present work we limit ourselves to the case that at least two of the three final mesons have the same mass. We thus define the variable $\vec{\rho}_{X}$ from the position vectors of the two mesons with equal masses and the variable $\vec{\lambda}_{X}$ from the other meson. For example, supposing that mesons $C_{1}\left(q_{1} \bar{q}_{4}\right)$ and $C_{2}\left(q_{2} \bar{q}_{1}\right)$ have equal masses, we define

$$
\vec{\rho}_{X}=\frac{1}{\sqrt{2}}\left(\vec{R}_{q_{1} \bar{q}_{4}}-\vec{R}_{q_{2} \bar{q}_{1}}\right)
$$




$$
\vec{\lambda}_{X}=\frac{1}{\sqrt{6}}\left(\vec{R}_{q_{1} \bar{q}_{4}}+\vec{R}_{q_{2} \bar{q}_{1}}-2 \vec{R}_{q_{3} \bar{q}_{2}}\right)
$$

which leads to

$$
d \vec{R}_{q_{1} \bar{q}_{4}} d \vec{R}_{q_{2} \bar{q}_{1}} d \vec{R}_{q_{3} \bar{q}_{2}}=3 \sqrt{3} d \vec{R}_{\text {total }} d \vec{\rho}_{X} d \vec{\lambda}_{X}
$$

From the mass $m_{C_{1}}$ of meson $C_{1}$, the mass $m_{C_{2}}$ of meson $C_{2}$, and the mass $m_{C_{3}}$ of meson $C_{3}$, we define

$$
\begin{aligned}
& m_{\rho}=m_{C_{1}}=m_{C_{2}}, \\
& m_{\lambda}=\frac{3 m_{C_{1}} m_{C_{3}}}{2 m_{C_{1}}+m_{C_{3}}} .
\end{aligned}
$$

Let $\vec{p}_{\rho_{X}}\left(\vec{p}_{\lambda_{X}}\right)$ be $m_{\rho}\left(m_{\lambda}\right)$ times the derivative of $\vec{\rho}_{X}\left(\vec{\lambda}_{X}\right)$ with respect to time. We then get

$$
\vec{P}_{q_{1} \bar{q}_{4}} \cdot \vec{R}_{q_{1} \bar{q}_{4}}+\vec{P}_{q_{2} \bar{q}_{1}} \cdot \vec{R}_{q_{2} \bar{q}_{1}}+\vec{P}_{q_{3} \bar{q}_{2}} \cdot \vec{R}_{q_{3} \bar{q}_{2}}=\vec{P}_{\mathrm{f}} \cdot \vec{R}_{\text {total }}+\vec{p}_{\rho_{X}} \cdot \vec{\rho}_{X}+\vec{p}_{\lambda_{X}} \cdot \vec{\lambda}_{X}
$$

In terms of $\vec{\rho}_{X}, \vec{\lambda}_{X}, \vec{p}_{\rho_{X}}$, and $\vec{p}_{\lambda_{X}}$, we have

$$
\begin{aligned}
\left\langle C_{1}, C_{2}, C_{3}\left|V_{\mathrm{D}_{1}}\right| A, B\right\rangle= & \frac{3 \sqrt{3}\left(m_{q_{1}}+m_{\bar{q}_{4}}\right)^{3}\left(m_{q_{2}}+m_{\bar{q}_{1}}\right)^{3}\left(m_{q_{3}}+m_{\bar{q}_{2}}\right)^{3}}{\sqrt{V^{5}}\left(m_{q_{1}} m_{q_{2}} m_{q_{3}}+m_{\bar{q}_{1}} m_{\bar{q}_{2}} m_{\bar{q}_{4}}\right)^{3}} \int d \vec{r}_{q_{1} \bar{q}_{1}} d \vec{r}_{q_{2} \bar{q}_{2}} d \vec{R}_{\text {total }} d \vec{\rho}_{X} d \vec{\lambda}_{X} \\
& \times \psi_{q_{1}}^{+} \bar{q}_{4}\left(\vec{r}_{q_{1} \bar{q}_{4}}\right) \psi_{q_{2}}^{+} \bar{q}_{1}\left(\vec{r}_{q_{2} \bar{q}_{1}}\right) \psi_{q_{3} \bar{q}_{2}}^{+}\left(\vec{r}_{q_{3} \bar{q}_{q_{2}}}\right) V_{\mathrm{D}_{1}} \psi_{q_{1} \bar{q}_{1}}\left(\vec{r}_{q_{1} \bar{q}_{1}}\right) \psi_{q_{2} \bar{q}_{2}}\left(\vec{r}_{q_{2} \bar{q}_{2}}\right) \\
& \times \exp \left(-i \vec{P}_{\mathrm{f}} \cdot \vec{R}_{\text {total }}-i \vec{p}_{\rho_{X}} \cdot \vec{\rho}_{X}-i \vec{p}_{\lambda_{X}} \cdot \vec{\lambda}_{X}+i \vec{P}_{\mathrm{i}} \cdot \vec{R}_{\text {total }}+i \vec{p}_{q_{1} \bar{q}_{1}, q_{2} \bar{q}_{2}} \cdot \vec{r}_{\left.q_{1} \bar{q}_{1}, q_{2} \bar{q}_{2}\right)}\right) \\
= & (2 \pi)^{3} \delta^{3}\left(\vec{P}_{\mathrm{i}}-\vec{P}_{\mathrm{f}}\right) \frac{\mathcal{M}_{\mathrm{D}_{1}}}{\sqrt{V^{5}} \sqrt{2 E_{A} 2 E_{B} 2 E_{C_{1}} 2 E_{C_{2}} 2 E_{C_{3}}}},
\end{aligned}
$$

where $\vec{r}_{q_{1} \bar{q}_{1}, q_{2} \bar{q}_{2}}$ and $\vec{p}_{q_{1} \bar{q}_{1}, q_{2} \bar{q}_{2}}$ are the relative coordinate and the relative momentum of $q_{1} \bar{q}_{1}$ and $q_{2} \bar{q}_{2}$, respectively. $\mathcal{M}_{\mathrm{D}_{1}}$ is the transition amplitude given by

$$
\begin{aligned}
\mathcal{M}_{\mathrm{D}_{1}}= & \sqrt{2 E_{A} 2 E_{B} 2 E_{C_{1}} 2 E_{C_{2}} 2 E_{C_{3}}} \frac{3 \sqrt{3}\left(m_{q_{1}}+m_{\bar{q}_{4}}\right)^{3}\left(m_{q_{2}}+m_{\bar{q}_{1}}\right)^{3}\left(m_{q_{3}}+m_{\bar{q}_{2}}\right)^{3}}{\left(m_{q_{1}} m_{q_{2}} m_{q_{3}}+m_{\bar{q}_{1}} m_{\bar{q}_{2}} m_{\bar{q}_{4}}\right)^{3}} \\
& \times \int d \vec{r}_{q_{1} \bar{q}_{1}} d \vec{r}_{q_{2} \bar{q}_{2}} d \vec{\rho}_{X} d \vec{\lambda}_{X} \psi_{q_{1} \bar{q}_{4}}^{+}\left(\vec{r}_{q_{1} \bar{q}_{4}}\right) \psi_{q_{2} \bar{q}_{1}}^{+}\left(\vec{r}_{q_{2} \bar{q}_{1}}\right) \psi_{q_{3} \bar{q}_{2}}^{+}\left(\vec{r}_{q_{3} \bar{q}_{2}}\right) V_{\mathrm{D}_{1}} \\
& \times \psi_{q_{1} \bar{q}_{1}}\left(\vec{r}_{q_{1} \bar{q}_{1}}\right) \psi_{q_{2} \bar{q}_{2}}\left(\vec{r}_{q_{2} \bar{q}_{2}}\right) \exp \left(-i \vec{p}_{\rho_{X}} \cdot \vec{\rho}_{X}-i \vec{p}_{\lambda_{X}} \cdot \vec{\lambda}_{X}+i \vec{p}_{q_{1} \bar{q}_{1}, q_{2} \bar{q}_{2}} \cdot \vec{r}_{q_{1} \bar{q}_{1}, q_{2} \bar{q}_{2}}\right) .
\end{aligned}
$$

For diagram $\mathrm{D}_{2}$ we have

$$
\left\langle C_{1}, C_{2}, C_{3}\left|V_{\mathrm{D}_{2}}\right| A, B\right\rangle=\left\langle q_{1} \bar{q}_{4}, q_{2} \bar{q}_{1}, q_{3} \bar{q}_{2}\left|V_{\mathrm{D}_{2}}\right| q_{1} \bar{q}_{1}, q_{2} \bar{q}_{2}\right\rangle=(2 \pi)^{3} \delta^{3}\left(\vec{P}_{\mathrm{i}}-\vec{P}_{\mathrm{f}}\right) \frac{\mathcal{M}_{\mathrm{D}_{2}}}{\sqrt{V^{5}} \sqrt{2 E_{A} 2 E_{B} 2 E_{C_{1}} 2 E_{C_{2}} 2 E_{C_{3}}}},
$$

where the transition amplitude $\mathcal{M}_{\mathrm{D}_{2}}$ is obtained from Eq. (19) by replacing $V_{\mathrm{D}_{1}}$ with $V_{\mathrm{D}_{2}}$. For diagram $\mathrm{D}_{3}$ we have

$$
\left\langle C_{1}, C_{2}, C_{3}\left|V_{\mathrm{D}_{3}}\right| A, B\right\rangle=\left\langle q_{1} \bar{q}_{4}, q_{2} \bar{q}_{1}, q_{3} \bar{q}_{2}\left|V_{\mathrm{D}_{3}}\right| q_{1} \bar{q}_{1}, q_{2} \bar{q}_{2}\right\rangle=(2 \pi)^{3} \delta^{3}\left(\vec{P}_{\mathrm{i}}-\vec{P}_{\mathrm{f}}\right) \frac{\mathcal{M}_{\mathrm{D}_{3}}}{\sqrt{V^{5}} \sqrt{2 E_{A} 2 E_{B} 2 E_{C_{1}} 2 E_{C_{2}} 2 E_{C_{3}}}},
$$

where the transition amplitude $\mathcal{M}_{\mathrm{D}_{3}}$ is obtained from Eq. (19) by replacing $V_{\mathrm{D}_{1}}$ with $V_{\mathrm{D}_{3}}$. For diagram $\mathrm{D}_{4}$ we have 


$$
\left\langle C_{1}, C_{2}, C_{3}\left|V_{\mathrm{D}_{4}}\right| A, B\right\rangle=\left\langle q_{1} \bar{q}_{4}, q_{2} \bar{q}_{1}, q_{3} \bar{q}_{2}\left|V_{\mathrm{D}_{4}}\right| q_{1} \bar{q}_{1}, q_{2} \bar{q}_{2}\right\rangle=(2 \pi)^{3} \delta^{3}\left(\vec{P}_{\mathrm{i}}-\vec{P}_{\mathrm{f}}\right) \frac{\mathcal{M}_{\mathrm{D}_{4}}}{\sqrt{V^{5}} \sqrt{2 E_{A} 2 E_{B} 2 E_{C_{1}} 2 E_{C_{2}} 2 E_{C_{3}}}},
$$

where the transition amplitude $\mathcal{M}_{\mathrm{D}_{4}}$ is obtained from Eq. (19) by replacing $V_{\mathrm{D}_{1}}$ with $V_{\mathrm{D}_{4}}$.

Next, we consider the four diagrams in Fig. 2. The five independent constituent position-vectors, $\vec{r}_{q_{1}}, \vec{r}_{\bar{q}_{1}}, \vec{r}_{q_{2}}, \vec{r}_{\bar{q}_{2}}$, and $\vec{r}_{q_{3}}$, are related to $\vec{r}_{q_{1} \bar{q}_{1}}, \vec{r}_{q_{2} \bar{q}_{2}}, \vec{R}_{q_{1} \bar{q}_{2}}, \vec{R}_{q_{2} \bar{q}_{4}}$, and $\vec{R}_{q_{3} \bar{q}_{1}}$ by

$$
\begin{aligned}
& \vec{r}_{q_{1}}=\frac{m_{\bar{q}_{1}} m_{\bar{q}_{2}} m_{\bar{q}_{4}}}{m_{q_{1}} m_{q_{2}} m_{q_{3}}+m_{\bar{q}_{1}} m_{\bar{q}_{2}} m_{\bar{q}_{4}}} \vec{r}_{q_{1} \bar{q}_{1}}+\frac{m_{q_{2}} m_{q_{3}} m_{\bar{q}_{2}}}{m_{q_{1}} m_{q_{2}} m_{q_{3}}+m_{\bar{q}_{1}} m_{\bar{q}_{2}} m_{\bar{q}_{4}}} \vec{r}_{q_{2} \bar{q}_{2}}+\frac{m_{q_{2}} m_{q_{3}}\left(m_{q_{1}}+m_{\bar{q}_{2}}\right)}{m_{q_{1}} m_{q_{2}} m_{q_{3}}+m_{\bar{q}_{1}} m_{\bar{q}_{2}} m_{\bar{q}_{4}}} \vec{R}_{q_{1} \bar{q}_{2}} \\
& -\frac{m_{q_{3}} m_{\bar{q}_{2}}\left(m_{q_{2}}+m_{\bar{q}_{4}}\right)}{m_{q_{1}} m_{q_{2}} m_{q_{3}}+m_{\bar{q}_{1}} m_{\bar{q}_{2}} m_{\bar{q}_{4}}} \vec{R}_{q_{2} \bar{q}_{4}}+\frac{m_{\bar{q}_{2}} m_{\bar{q}_{4}}\left(m_{q_{3}}+m_{\bar{q}_{1}}\right)}{m_{q_{1}} m_{q_{2}} m_{q_{3}}+m_{\bar{q}_{1}} m_{\bar{q}_{2}} m_{\bar{q}_{4}}} \vec{R}_{q_{3} \bar{q}_{1}}, \\
& \vec{r}_{\bar{q}_{1}}=-\frac{m_{q_{1}} m_{q_{2}} m_{q_{3}}}{m_{q_{1}} m_{q_{2}} m_{q_{3}}+m_{\bar{q}_{1}} m_{\bar{q}_{2}} m_{\bar{q}_{4}}} \vec{r}_{q_{1} \bar{q}_{1}}+\frac{m_{q_{2}} m_{q_{3}} m_{\bar{q}_{2}}}{m_{q_{1}} m_{q_{2}} m_{q_{3}}+m_{\bar{q}_{1}} m_{\bar{q}_{2}} m_{\bar{q}_{4}}} \vec{r}_{q_{2} \bar{q}_{2}}+\frac{m_{q_{2}} m_{q_{3}}\left(m_{q_{1}}+m_{\bar{q}_{2}}\right)}{m_{q_{1}} m_{q_{2}} m_{q_{3}}+m_{\bar{q}_{1}} m_{\bar{q}_{2}} m_{\bar{q}_{4}}} \vec{R}_{q_{1} \bar{q}_{2}} \\
& -\frac{m_{q_{3}} m_{\bar{q}_{2}}\left(m_{q_{2}}+m_{\bar{q}_{4}}\right)}{m_{q_{1}} m_{q_{2}} m_{q_{3}}+m_{\bar{q}_{1}} m_{\bar{q}_{2}} m_{\bar{q}_{4}}} \vec{R}_{q_{2} \bar{q}_{4}}+\frac{m_{\bar{q}_{2}} m_{\bar{q}_{4}}\left(m_{q_{3}}+m_{\bar{q}_{1}}\right)}{m_{q_{1}} m_{q_{2}} m_{q_{3}}+m_{\bar{q}_{1}} m_{\bar{q}_{2}} m_{\bar{q}_{4}}} \vec{R}_{q_{3} \bar{q}_{1}}, \\
& \vec{r}_{q_{2}}=-\frac{m_{q_{1}} m_{\bar{q}_{1}} m_{\bar{q}_{4}}}{m_{q_{1}} m_{q_{2}} m_{q_{3}}+m_{\bar{q}_{1}} m_{\bar{q}_{2}} m_{\bar{q}_{4}}} \vec{r}_{q_{1} \bar{q}_{1}}+\frac{m_{\bar{q}_{1}} m_{\bar{q}_{2}} m_{\bar{q}_{4}}}{m_{q_{1}} m_{q_{2}} m_{q_{3}}+m_{\bar{q}_{1}} m_{\bar{q}_{2}} m_{\bar{q}_{4}}} \vec{r}_{q_{2} \bar{q}_{2}}+\frac{m_{\bar{q}_{1}} m_{\bar{q}_{4}}\left(m_{q_{1}}+m_{\bar{q}_{2}}\right)}{m_{q_{1}} m_{q_{2}} m_{q_{3}}+m_{\bar{q}_{1}} m_{\bar{q}_{2}} m_{\bar{q}_{4}}} \vec{R}_{q_{1} \bar{q}_{2}} \\
& +\frac{m_{q_{1}} m_{q_{3}}\left(m_{q_{2}}+m_{\bar{q}_{4}}\right)}{m_{q_{1}} m_{q_{2}} m_{q_{3}}+m_{\bar{q}_{1}} m_{\bar{q}_{2}} m_{\bar{q}_{4}}} \vec{R}_{q_{2} \bar{q}_{4}}-\frac{m_{q_{1}} m_{\bar{q}_{4}}\left(m_{q_{3}}+m_{\bar{q}_{1}}\right)}{m_{q_{1}} m_{q_{2}} m_{q_{3}}+m_{\bar{q}_{1}} m_{\bar{q}_{2}} m_{\bar{q}_{4}}} \vec{R}_{q_{3} \bar{q}_{1}}, \\
& \vec{r}_{\bar{q}_{2}}=-\frac{m_{q_{1}} m_{\bar{q}_{1}} m_{\bar{q}_{4}}}{m_{q_{1}} m_{q_{2}} m_{q_{3}}+m_{\bar{q}_{1}} m_{\bar{q}_{2}} m_{\bar{q}_{4}}} \vec{r}_{q_{1} \bar{q}_{1}}-\frac{m_{q_{1}} m_{q_{2}} m_{q_{3}}}{m_{q_{1}} m_{q_{2}} m_{q_{3}}+m_{\bar{q}_{1}} m_{\bar{q}_{2}} m_{\bar{q}_{4}}} \vec{r}_{q_{2} \bar{q}_{2}}+\frac{m_{\bar{q}_{1}} m_{\bar{q}_{4}}\left(m_{q_{1}}+m_{\bar{q}_{2}}\right)}{m_{q_{1}} m_{q_{2}} m_{q_{3}}+m_{\bar{q}_{1}} m_{\bar{q}_{2}} m_{\bar{q}_{4}}} \vec{R}_{q_{1} \bar{q}_{2}} \\
& +\frac{m_{q_{1}} m_{q_{3}}\left(m_{q_{2}}+m_{\bar{q}_{4}}\right)}{m_{q_{1}} m_{q_{2}} m_{q_{3}}+m_{\bar{q}_{1}} m_{\bar{q}_{2}} m_{\bar{q}_{4}}} \vec{R}_{q_{2} \bar{q}_{4}}-\frac{m_{q_{1}} m_{\bar{q}_{4}}\left(m_{q_{3}}+m_{\bar{q}_{1}}\right)}{m_{q_{1}} m_{q_{2}} m_{q_{3}}+m_{\bar{q}_{1}} m_{\bar{q}_{2}} m_{\bar{q}_{4}}} \vec{R}_{q_{3} \bar{q}_{1}}, \\
& \vec{r}_{q_{3}}=\frac{m_{q_{1}} m_{q_{2}} m_{\bar{q}_{1}}}{m_{q_{1}} m_{q_{2}} m_{q_{3}}+m_{\bar{q}_{1}} m_{\bar{q}_{2}} m_{\bar{q}_{4}}} \vec{r}_{q_{1} \bar{q}_{1}}-\frac{m_{q_{2}} m_{\bar{q}_{1}} m_{\bar{q}_{2}}}{m_{q_{1}} m_{q_{2}} m_{q_{3}}+m_{\bar{q}_{1}} m_{\bar{q}_{2}} m_{\bar{q}_{4}}} \vec{r}_{q_{2} \bar{q}_{2}}-\frac{m_{q_{2}} m_{\bar{q}_{1}}\left(m_{q_{1}}+m_{\bar{q}_{2}}\right)}{m_{q_{1}} m_{q_{2}} m_{q_{3}}+m_{\bar{q}_{1}} m_{\bar{q}_{2}} m_{\bar{q}_{4}}} \vec{R}_{q_{1} \bar{q}_{2}} \\
& +\frac{m_{\bar{q}_{1}} m_{\bar{q}_{2}}\left(m_{q_{2}}+m_{\bar{q}_{4}}\right)}{m_{q_{1}} m_{q_{2}} m_{q_{3}}+m_{\bar{q}_{1}} m_{\bar{q}_{2}} m_{\bar{q}_{4}}} \vec{R}_{q_{2} \bar{q}_{4}}+\frac{m_{q_{1}} m_{q_{2}}\left(m_{q_{3}}+m_{\bar{q}_{1}}\right)}{m_{q_{1}} m_{q_{2}} m_{q_{3}}+m_{\bar{q}_{1}} m_{\bar{q}_{2}} m_{\bar{q}_{4}}} \vec{R}_{q_{3} \bar{q}_{1}},
\end{aligned}
$$

which lead to

$$
d \vec{r}_{q_{1}} d \vec{r}_{\bar{q}_{1}} d \vec{r}_{q_{2}} d \vec{r}_{\bar{q}_{2}} d \vec{r}_{q_{3}}=\frac{\left(m_{q_{1}}+m_{\bar{q}_{2}}\right)^{3}\left(m_{q_{2}}+m_{\bar{q}_{4}}\right)^{3}\left(m_{q_{3}}+m_{\bar{q}_{1}}\right)^{3}}{\left(m_{q_{1}} m_{q_{2}} m_{q_{3}}+m_{\bar{q}_{1}} m_{\bar{q}_{2}} m_{\bar{q}_{4}}\right)^{3}} d \vec{r}_{q_{1} \bar{q}_{1}} d \vec{r}_{q_{2} \bar{q}_{2}} d \vec{R}_{q_{1} \bar{q}_{2}} d \vec{R}_{q_{2} \bar{q}_{4}} d \vec{R}_{q_{3} \bar{q}_{1}} .
$$


From the transition potential $V_{\mathrm{D}_{5}}$ and the wave functions of the initial and final mesons, we have for diagram $\mathrm{D}_{5}$ in Fig. 2 :

$$
\begin{aligned}
& \left\langle C_{1}, C_{2}, C_{3}\left|V_{\mathrm{D}_{5}}\right| A, B\right\rangle=\left\langle q_{1} \bar{q}_{2}, q_{2} \bar{q}_{4}, q_{3} \bar{q}_{1}\left|V_{\mathrm{D}_{5}}\right| q_{1} \bar{q}_{1}, q_{2} \bar{q}_{2}\right\rangle \\
& =\int d \vec{r}_{q_{1}} d \vec{r}_{\bar{q}_{1}} d \vec{r}_{q_{2}} d \vec{r}_{\bar{q}_{2}} d \vec{r}_{q_{3}} \frac{e^{-i \vec{P}_{q_{1} \bar{q}_{2}} \cdot \vec{R}_{q_{1}} \bar{q}_{2}}}{\sqrt{V}} \psi_{q_{1} \bar{q}_{2}}^{+}\left(\vec{r}_{q_{1} \bar{q}_{2}}\right) \frac{e^{-i \vec{P}_{q_{2} \bar{q}} \cdot \vec{R}_{q_{2} \bar{q}_{4}}}}{\sqrt{V}} \psi_{q_{2} \bar{q}_{4}}^{+}\left(\vec{r}_{q_{2} \bar{q}_{4}}\right) \\
& \times \frac{e^{-i \vec{P}_{q_{3} \bar{q}_{1}} \cdot \vec{R}_{q_{3} \bar{q}_{1}}}}{\sqrt{V}} \psi_{q_{3} \bar{q}_{1}}^{+}\left(\vec{r}_{q_{3} \bar{q}_{1}}\right) V_{\mathrm{D}_{5}} \frac{e^{i \vec{P}_{q_{1}} \cdot \bar{q}_{1} \cdot \vec{R}_{q_{1}} \bar{q}_{1}}}{\sqrt{V}} \psi_{q_{1} \bar{q}_{1}}\left(\vec{r}_{q_{1} \bar{q}_{1}}\right) \frac{e^{i \vec{P}_{q_{2} \bar{q}_{2}} \cdot \vec{R}_{q_{2}} \bar{q}_{2}}}{\sqrt{V}} \psi_{q_{2} \bar{q}_{2}}\left(\vec{r}_{q_{2} \bar{q}_{2}}\right) \\
& =\frac{\left(m_{q_{1}}+m_{\bar{q}_{2}}\right)^{3}\left(m_{q_{2}}+m_{\bar{q}_{4}}\right)^{3}\left(m_{q_{3}}+m_{\bar{q}_{1}}\right)^{3}}{\sqrt{V^{5}}\left(m_{q_{1}} m_{q_{2}} m_{q_{3}}+m_{\bar{q}_{1}} m_{\bar{q}_{2}} m_{\bar{q}_{4}}\right)^{3}} \int d \vec{r}_{q_{1} \bar{q}_{1}} d \vec{r}_{q_{2} \bar{q}_{2}} d \vec{R}_{q_{1} \bar{q}_{2}} d \vec{R}_{q_{2} \bar{q}_{4}} d \vec{R}_{q_{3} \bar{q}_{1}} \\
& \times \psi_{q_{1} \bar{q}_{2}}^{+}\left(\vec{r}_{q_{1} \bar{q}_{2}}\right) \psi_{q_{2} \bar{q}_{4}}^{+}\left(\vec{r}_{q_{2} \bar{q}_{4}}\right) \psi_{q_{3} \bar{q}_{1}}^{+}\left(\vec{r}_{q_{3} \bar{q}_{1}}\right) V_{\mathrm{D}_{5}} \psi_{q_{1} \bar{q}_{1}}\left(\vec{r}_{q_{1} \bar{q}_{1}}\right) \psi_{q_{2} \bar{q}_{2}}\left(\vec{r}_{q_{2} \bar{q}_{2}}\right) \\
& \times \exp \left(-i \vec{P}_{q_{1} \bar{q}_{2}} \cdot \vec{R}_{q_{1} \bar{q}_{2}}-i \vec{P}_{q_{2} \bar{q}_{4}} \cdot \vec{R}_{q_{2} \bar{q}_{4}}-i \vec{P}_{q_{3} \bar{q}_{1}} \cdot \vec{R}_{q_{3} \bar{q}_{1}}+i \vec{P}_{q_{1} \bar{q}_{1}} \cdot \vec{R}_{q_{1} \bar{q}_{1}}+i \vec{P}_{q_{2} \bar{q}_{2}} \cdot \vec{R}_{q_{2} \bar{q}_{2}}\right) \\
& =\frac{3 \sqrt{3}\left(m_{q_{1}}+m_{\bar{q}_{2}}\right)^{3}\left(m_{q_{2}}+m_{\bar{q}_{4}}\right)^{3}\left(m_{q_{3}}+m_{\bar{q}_{1}}\right)^{3}}{\sqrt{V^{5}}\left(m_{q_{1}} m_{q_{2}} m_{q_{3}}+m_{\bar{q}_{1}} m_{\bar{q}_{2}} m_{\bar{q}_{4}}\right)^{3}} \int d \vec{r}_{q_{1} \bar{q}_{1}} d \vec{r}_{q_{2} \bar{q}_{2}} d \vec{R}_{\text {total }} d \vec{\rho}_{Y} d \vec{\lambda}_{Y} \\
& \times \psi_{q_{1} \bar{q}_{2}}^{+}\left(\vec{r}_{q_{1} \bar{q}_{2}}\right) \psi_{q_{2} \bar{q}_{4}}^{+}\left(\vec{r}_{q_{2} \bar{q}_{4}}\right) \psi_{q_{3} \bar{q}_{1}}^{+}\left(\vec{r}_{q_{3} \bar{q}_{1}}\right) V_{\mathrm{D}_{5}} \psi_{q_{1} \bar{q}_{1}}\left(\vec{r}_{q_{1} \bar{q}_{1}}\right) \psi_{q_{2} \bar{q}_{2}}\left(\vec{r}_{q_{2} \bar{q}_{2}}\right) \\
& \times \exp \left(-i \vec{P}_{\mathrm{f}} \cdot \vec{R}_{\text {total }}-i \vec{p}_{\rho_{Y}} \cdot \vec{\rho}_{Y}-i \vec{p}_{\lambda_{Y}} \cdot \vec{\lambda}_{Y}+i \vec{P}_{\mathrm{i}} \cdot \vec{R}_{\text {total }}+i \vec{p}_{q_{1} \bar{q}_{1}, q_{2} \bar{q}_{2}} \cdot \vec{r}_{q_{1} \bar{q}_{1}, q_{2} \bar{q}_{2}}\right) \\
& =(2 \pi)^{3} \delta^{3}\left(\vec{P}_{\mathrm{i}}-\vec{P}_{\mathrm{f}}\right) \frac{\mathcal{M}_{\mathrm{D}_{5}}}{\sqrt{V^{5}} \sqrt{2 E_{A} 2 E_{B} 2 E_{C_{1}} 2 E_{C_{2}} 2 E_{C_{3}}}},
\end{aligned}
$$

where $\mathcal{M}_{\mathrm{D}_{5}}$ is the transition amplitude given by

$$
\begin{aligned}
\mathcal{M}_{\mathrm{D}_{5}}= & \sqrt{2 E_{A} 2 E_{B} 2 E_{C_{1}} 2 E_{C_{2}} 2 E_{C_{3}}} \frac{3 \sqrt{3}\left(m_{q_{1}}+m_{\bar{q}_{2}}\right)^{3}\left(m_{q_{2}}+m_{\bar{q}_{4}}\right)^{3}\left(m_{q_{3}}+m_{\bar{q}_{1}}\right)^{3}}{\left(m_{q_{1}} m_{q_{2}} m_{q_{3}}+m_{\bar{q}_{1}} m_{\bar{q}_{2}} m_{\bar{q}_{4}}\right)^{3}} \\
& \times \int d \vec{r}_{q_{1} \bar{q}_{1}} d \vec{r}_{q_{2} \bar{q}_{2}} d \vec{\rho}_{Y} d \vec{\lambda}_{Y} \psi_{q_{1} \bar{q}_{2}}^{+}\left(\vec{r}_{q_{1} \bar{q}_{2}}\right) \psi_{q_{2} \bar{q}_{4}}^{+}\left(\vec{r}_{q_{2} \bar{q}_{4}}\right) \psi_{q_{3} \bar{q}_{1}}^{+}\left(\vec{r}_{q_{3} \bar{q}_{1}}\right) V_{\mathrm{D}_{5}} \\
& \times \psi_{q_{1} \bar{q}_{1}}\left(\vec{r}_{q_{1} \bar{q}_{1}}\right) \psi_{q_{2} \bar{q}_{2}}\left(\vec{r}_{q_{2}} \bar{q}_{2}\right) \exp \left(-i \vec{p}_{\rho_{Y}} \cdot \vec{\rho}_{Y}-i \vec{p}_{\lambda_{Y}} \cdot \vec{\lambda}_{Y}+i \vec{p}_{q_{1} \bar{q}_{1}, q_{2} \bar{q}_{2}} \cdot \vec{r}_{q_{1} \bar{q}_{1}, q_{2} \bar{q}_{2}}\right) .
\end{aligned}
$$

In the above two equations the variable $\vec{\rho}_{Y}$ is defined from the position vectors of the two mesons with equal masses, and the variable $\vec{\lambda}_{Y}$ from the other meson. In case that mesons $C_{1}\left(q_{1} \bar{q}_{2}\right)$ and $C_{2}\left(q_{2} \bar{q}_{4}\right)$ have the same mass, we define

$$
\begin{gathered}
\vec{\rho}_{Y}=\frac{1}{\sqrt{2}}\left(\vec{R}_{q_{1} \bar{q}_{2}}-\vec{R}_{q_{2} \bar{q}_{4}}\right), \\
\vec{\lambda}_{Y}=\frac{1}{\sqrt{6}}\left(\vec{R}_{q_{1} \bar{q}_{2}}+\vec{R}_{q_{2} \bar{q}_{4}}-2 \vec{R}_{q_{3} \bar{q}_{1}}\right) .
\end{gathered}
$$

Let $\vec{p}_{\rho_{Y}}\left(\vec{p}_{\lambda_{Y}}\right)$ be $m_{\rho}\left(m_{\lambda}\right)$ times the derivative of $\vec{\rho}_{Y}\left(\vec{\lambda}_{Y}\right)$ with respect to time. In Eq. (29) we have used the two equalities,

$$
\begin{gathered}
d \vec{R}_{q_{1} \bar{q}_{2}} d \vec{R}_{q_{2} \bar{q}_{4}} d \vec{R}_{q_{3} \bar{q}_{1}}=3 \sqrt{3} d \vec{R}_{\text {total }} d \vec{\rho}_{Y} d \vec{\lambda}_{Y}, \\
\vec{P}_{q_{1} \bar{q}_{2}} \cdot \vec{R}_{q_{1} \bar{q}_{2}}+\vec{P}_{q_{2} \bar{q}_{4}} \cdot \vec{R}_{q_{2} \bar{q}_{4}}+\vec{P}_{q_{3} \bar{q}_{1}} \cdot \vec{R}_{q_{3} \bar{q}_{1}}=\vec{P}_{\mathrm{f}} \cdot \vec{R}_{\text {total }}+\vec{p}_{\rho_{Y}} \cdot \vec{\rho}_{Y}+\vec{p}_{\lambda_{Y}} \cdot \vec{\lambda}_{Y} .
\end{gathered}
$$

For diagram $\mathrm{D}_{6}$ we have

$$
\left\langle C_{1}, C_{2}, C_{3}\left|V_{\mathrm{D}_{6}}\right| A, B\right\rangle=\left\langle q_{1} \bar{q}_{2}, q_{2} \bar{q}_{4}, q_{3} \bar{q}_{1}\left|V_{\mathrm{D}_{6}}\right| q_{1} \bar{q}_{1}, q_{2} \bar{q}_{2}\right\rangle=(2 \pi)^{3} \delta^{3}\left(\vec{P}_{\mathrm{i}}-\vec{P}_{\mathrm{f}}\right) \frac{\mathcal{M}_{\mathrm{D}_{6}}}{\sqrt{V^{5}} \sqrt{2 E_{A} 2 E_{B} 2 E_{C_{1}} 2 E_{C_{2}} 2 E_{C_{3}}}},
$$

where the transition amplitude $\mathcal{M}_{\mathrm{D}_{6}}$ is obtained from Eq. (30) by replacing $V_{\mathrm{D}_{5}}$ with $V_{\mathrm{D}_{6}}$. For diagram $\mathrm{D}_{7}$ we have 


$$
\left\langle C_{1}, C_{2}, C_{3}\left|V_{\mathrm{D}_{7}}\right| A, B\right\rangle=\left\langle q_{1} \bar{q}_{2}, q_{2} \bar{q}_{4}, q_{3} \bar{q}_{1}\left|V_{\mathrm{D}_{7}}\right| q_{1} \bar{q}_{1}, q_{2} \bar{q}_{2}\right\rangle=(2 \pi)^{3} \delta^{3}\left(\vec{P}_{\mathrm{i}}-\vec{P}_{\mathrm{f}}\right) \frac{\mathcal{M}_{\mathrm{D}_{7}}}{\sqrt{V^{5}} \sqrt{2 E_{A} 2 E_{B} 2 E_{C_{1}} 2 E_{C_{2}} 2 E_{C_{3}}}},
$$

where the transition amplitude $\mathcal{M}_{\mathrm{D}_{7}}$ is obtained from Eq. (30) by replacing $V_{\mathrm{D}_{5}}$ with $V_{\mathrm{D}_{7}}$. For diagram $\mathrm{D}_{8}$ we have

$$
\left\langle C_{1}, C_{2}, C_{3}\left|V_{\mathrm{D}_{8}}\right| A, B\right\rangle=\left\langle q_{1} \bar{q}_{2}, q_{2} \bar{q}_{4}, q_{3} \bar{q}_{1}\left|V_{\mathrm{D}_{8}}\right| q_{1} \bar{q}_{1}, q_{2} \bar{q}_{2}\right\rangle=(2 \pi)^{3} \delta^{3}\left(\vec{P}_{\mathrm{i}}-\vec{P}_{\mathrm{f}}\right) \frac{\mathcal{M}_{\mathrm{D}_{8}}}{\sqrt{V^{5}} \sqrt{2 E_{A} 2 E_{B} 2 E_{C_{1}} 2 E_{C_{2}} 2 E_{C_{3}}}},
$$

where the transition amplitude $\mathcal{M}_{\mathrm{D}_{8}}$ is obtained from Eq. (30) by replacing $V_{\mathrm{D}_{5}}$ with $V_{\mathrm{D}_{8}}$.

Let $P_{A}\left(P_{B}\right)$ and $m_{A}\left(m_{B}\right)$ be the four-momentum and the mass of meson $A(B)$, respectively, and we have the Mandelstam variable $s=\left(P_{A}+P_{B}\right)^{2}$. Along the general lines provided in Ref. [59] on deriving the cross section from the transition amplitude, we get the unpolarized cross section for $A+B \rightarrow C_{1}+C_{2}+C_{3}$,

$$
\begin{aligned}
& \sigma^{\text {unpol }}=\frac{(2 \pi)^{4}}{4 \sqrt{\left(P_{A} \cdot P_{B}\right)^{2}-m_{A}^{2} m_{B}^{2}}} \frac{1}{\left(2 J_{A}+1\right)\left(2 J_{B}+1\right)} \int \frac{d^{3} P_{C_{1}}}{(2 \pi)^{3} 2 E_{C_{1}}} \frac{d^{3} P_{C_{2}}}{(2 \pi)^{3} 2 E_{C_{2}}} \frac{d^{3} P_{C_{3}}}{(2 \pi)^{3} 2 E_{C_{3}}} \delta\left(E_{\mathrm{f}}-E_{\mathrm{i}}\right) \delta^{3}\left(\vec{P}_{\mathrm{f}}-\vec{P}_{\mathrm{i}}\right) \\
& \times \sum_{J_{A z} J_{B z} J_{C_{1} z} J_{C_{2} z} J_{C_{3} z}}\left|\mathcal{M}_{\mathrm{D}_{1}}+\mathcal{M}_{\mathrm{D}_{2}}+\mathcal{M}_{\mathrm{D}_{3}}+\mathcal{M}_{\mathrm{D}_{4}}+\mathcal{M}_{\mathrm{D}_{5}}+\mathcal{M}_{\mathrm{D}_{6}}+\mathcal{M}_{\mathrm{D}_{7}}+\mathcal{M}_{\mathrm{D}_{8}}\right|^{2} \text {, }
\end{aligned}
$$

where $J_{i}\left(i=A, B, C_{1}, C_{2}, C_{3}\right)$ is the angular momentum of meson $i$ with the magnetic projection quantum number $J_{i z}$. With the equality $\left(P_{A} \cdot P_{B}\right)^{2}-m_{A}^{2} m_{B}^{2}=0.25\left[s-\left(m_{A}+m_{B}\right)^{2}\right]\left[s-\left(m_{A}-m_{B}\right)^{2}\right]$, integration over $\vec{P}_{C_{2}}$ leads to

$$
\begin{aligned}
& \sigma^{\mathrm{unpol}}=\frac{1}{16(2 \pi)^{5} \sqrt{\left[s-\left(m_{A}+m_{B}\right)^{2}\right]\left[s-\left(m_{A}-m_{B}\right)^{2}\right]}} \frac{1}{\left(2 J_{A}+1\right)\left(2 J_{B}+1\right)} \\
& \times \int \frac{d^{3} P_{C_{1}} d^{3} P_{C_{3}}}{E_{C_{1}} E_{C_{2}} E_{C_{3}}} \delta\left(E_{\mathrm{f}}-E_{\mathrm{i}}\right) \sum_{J_{A_{z}} J_{B_{z}} J_{C_{1} J^{z}} J_{C_{2} J^{z}} J_{C_{3} z}}\left|\mathcal{M}_{\mathrm{D}_{1}}+\mathcal{M}_{\mathrm{D}_{2}}+\mathcal{M}_{\mathrm{D}_{3}}+\mathcal{M}_{\mathrm{D}_{4}}+\mathcal{M}_{\mathrm{D}_{5}}+\mathcal{M}_{\mathrm{D}_{6}}+\mathcal{M}_{\mathrm{D}_{7}}+\mathcal{M}_{\mathrm{D}_{8}}\right|^{2} .
\end{aligned}
$$

Integration over $\left|\vec{P}_{C_{1}}\right|$ yields

$$
\begin{aligned}
& \sigma^{\mathrm{unpol}}=\frac{1}{16(2 \pi)^{5} \sqrt{\left[s-\left(m_{A}+m_{B}\right)^{2}\right]\left[s-\left(m_{A}-m_{B}\right)^{2}\right]}} \frac{1}{\left(2 J_{A}+1\right)\left(2 J_{B}+1\right)} \\
& \times \int \frac{d^{3} P_{C_{3}}}{E_{C_{3}}} d \Omega_{C_{1}} \frac{\left|\vec{P}_{C_{1}}\right|_{0}^{2}}{\left.|| \vec{P}_{C_{1}}\right|_{0} E_{C_{2}}+\left(\left|\vec{P}_{C_{1}}\right|_{0}-\left|\vec{P}_{A}+\vec{P}_{B}-\vec{P}_{C_{3}}\right| \cos \Theta\right) E_{C_{1}} \mid} \\
& \times \sum_{J_{A z} J_{B z} J_{C_{1} z} J_{C_{2} z} J_{C_{3} z}}\left|\mathcal{M}_{\mathrm{D}_{1}}+\mathcal{M}_{\mathrm{D}_{2}}+\mathcal{M}_{\mathrm{D}_{3}}+\mathcal{M}_{\mathrm{D}_{4}}+\mathcal{M}_{\mathrm{D}_{5}}+\mathcal{M}_{\mathrm{D}_{6}}+\mathcal{M}_{\mathrm{D}_{7}}+\mathcal{M}_{\mathrm{D}_{8}}\right|^{2},
\end{aligned}
$$

where $\Theta$ is the angle between $\vec{P}_{C_{1}}$ and $\vec{P}_{A}+\vec{P}_{B}-\vec{P}_{C_{3}}$, $d \Omega_{C_{1}}$ is the solid angle centered about the direction of $\vec{P}_{C_{1}}$, and $\left|\vec{P}_{C_{1}}\right|_{0}$ is the absolute value of $\vec{P}_{C_{1}}$ that satisfies $E_{\mathrm{f}}-E_{\mathrm{i}}=0$. The unpolarized cross section is a function of $\sqrt{s}$, which is the total energy of the two initial mesons in the center-of-mass frame.

\section{TRANSITION POTENTIAL}

In Fig. 3 the left diagram denotes the process $q^{\prime}\left(p_{1}\right) \rightarrow q^{\prime}\left(p_{1}^{\prime}\right)+q\left(p_{3}\right)+\bar{q}\left(-p_{4}\right)$, and the right diagram $\bar{q}^{\prime}\left(-p_{1}\right) \rightarrow \bar{q}^{\prime}\left(-p_{1}^{\prime}\right)+q\left(p_{3}\right)+\bar{q}\left(-p_{4}\right)$. In each diagram the wavy line represents the gluon which has four-momentum $k$, the color index $e$, and the space-time index $\tau$. Each vertex

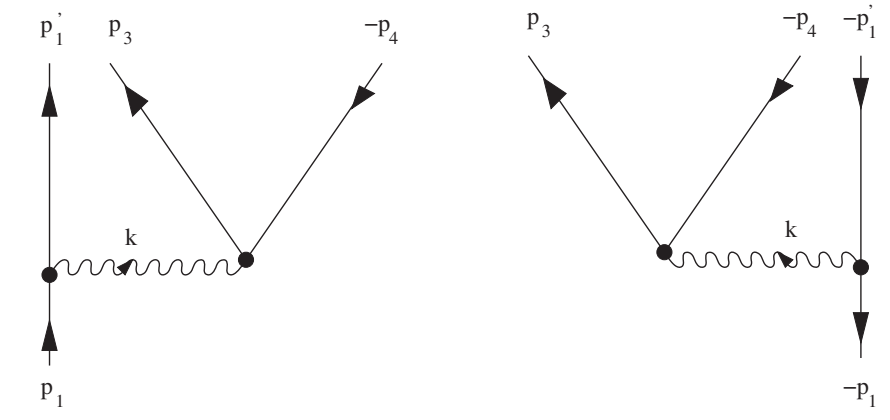

FIG. 3. Left diagram with $q^{\prime}\left(p_{1}\right) \rightarrow q^{\prime}\left(p_{1}^{\prime}\right)+q\left(p_{3}\right)+\bar{q}\left(-p_{4}\right)$ and right diagram with $\bar{q}^{\prime}\left(-p_{1}\right) \rightarrow \bar{q}^{\prime}\left(-p_{1}^{\prime}\right)+q\left(p_{3}\right)+\bar{q}\left(-p_{4}\right)$. 
involves the gauge coupling constant $g_{\mathrm{s}}$, the $S U(3)$ color generators $T^{e}(e=1, \ldots, 8)$, and the Dirac matrices $\gamma^{\tau}$. According to the Feynman rules in QCD [60], the amplitude for the left diagram in Fig. 3 is written as

$$
\begin{aligned}
\mathcal{M}_{\mathrm{c} q^{\prime} q \bar{q}}= & \frac{g_{s}^{2}}{k^{2}} \bar{\psi}_{q^{\prime}}\left(\vec{p}_{1}^{\prime}, s_{q^{\prime} z}^{\prime}\right) \gamma_{\tau} T^{e} \psi_{q^{\prime}}\left(\vec{p}_{1}, s_{q^{\prime} z}\right) \\
& \times \bar{\psi}_{q}\left(\vec{p}_{3}, s_{q z}\right) \gamma^{\tau} T^{e} \psi_{\bar{q}}\left(\vec{p}_{4}, s_{\bar{q} z}\right),
\end{aligned}
$$

and the amplitude for the right diagram is

$$
\begin{aligned}
\mathcal{M}_{\mathrm{c} \bar{q}^{\prime} q \bar{q}}= & -\frac{g_{s}^{2}}{k^{2}} \bar{\psi}_{\bar{q}^{\prime}}\left(\vec{p}_{1}, s_{\bar{q}^{\prime} z}\right) \gamma_{\tau} T^{e} \psi_{\bar{q}^{\prime}}\left(\vec{p}_{1}^{\prime}, s_{\bar{q}^{\prime} z}^{\prime}\right) \\
& \times \bar{\psi}_{q}\left(\vec{p}_{3}, s_{q z}\right) \gamma^{\tau} T^{e} \psi_{\bar{q}}\left(\vec{p}_{4}, s_{\bar{q}_{z}}\right),
\end{aligned}
$$

where repeated color and space-time indices ( $e$ and $\tau$ ) are summed. The quark spinors $\left(\psi_{q^{\prime}}\left(\vec{p}_{1}, s_{q^{\prime} z}\right), \psi_{q^{\prime}}\left(\vec{p}_{1}^{\prime}, s_{q^{\prime} z}^{\prime}\right)\right.$, $\left.\psi_{q}\left(\vec{p}_{3}, s_{q z}\right)\right)$ and the antiquark spinors $\left(\psi_{\bar{q}}\left(\vec{p}_{4}, s_{\bar{q}_{z}}\right)\right.$, $\left.\psi_{\bar{q}^{\prime}}\left(\vec{p}_{1}, s_{\bar{q}^{\prime} z}\right), \psi_{\bar{q}^{\prime}}\left(\vec{p}_{1}^{\prime}, s_{\bar{q}^{\prime} z}^{\prime}\right)\right)$ are given by $[53,59]$

$$
\begin{aligned}
& \psi_{q}\left(\vec{p}_{3}, s_{q z}\right)=\left(\begin{array}{c}
G_{3}\left(\vec{p}_{3}\right) \\
\frac{\vec{\sigma} \cdot \vec{p}_{3}}{2 m_{q}} G_{3}\left(\vec{p}_{3}\right)
\end{array}\right) \chi_{s_{q z}}, \\
& \psi_{\bar{q}}\left(\vec{p}_{4}, s_{\bar{q} z}\right)=\left(\begin{array}{c}
\frac{\vec{\sigma} \cdot \vec{p}_{4}}{2 m_{\bar{q}}} G_{4}\left(\vec{p}_{4}\right) \\
G_{4}\left(\vec{p}_{4}\right)
\end{array}\right) \chi_{s_{\bar{q} \bar{z}}},
\end{aligned}
$$

$$
\begin{aligned}
& \psi_{q^{\prime}}\left(\vec{p}_{1}, s_{q^{\prime} z}\right)=\left(\begin{array}{c}
G_{1}\left(\vec{p}_{1}\right) \\
\frac{\vec{\sigma} \cdot \vec{p}_{1}}{2 m_{q^{\prime}}} G_{1}\left(\vec{p}_{1}\right)
\end{array}\right) \chi_{s_{q^{\prime} z^{\prime}},}, \\
& \psi_{q^{\prime}}\left(\vec{p}_{1}^{\prime}, s_{q^{\prime} z}^{\prime}\right)=\left(\begin{array}{c}
G_{1}^{\prime}\left(\vec{p}_{1}^{\prime}\right) \\
\frac{\vec{\sigma} \cdot \vec{p}_{1}^{\prime}}{2 m_{q^{\prime}}} G_{1}^{\prime}\left(\vec{p}_{1}^{\prime}\right)
\end{array}\right) \chi_{s_{q^{\prime} z^{\prime}},}, \\
& \psi_{\bar{q}^{\prime}}\left(\vec{p}_{1}, s_{\bar{q}^{\prime} z}\right)=\left(\begin{array}{c}
\frac{\vec{\sigma} \cdot \vec{p}_{1}}{2 m_{\bar{q}^{\prime}}} G_{1}\left(\vec{p}_{1}\right) \\
G_{1}\left(\vec{p}_{1}\right)
\end{array}\right) \chi_{s_{\bar{q}^{\prime} z},}, \\
& \psi_{\bar{q}^{\prime}}\left(\vec{p}_{1}^{\prime}, s_{\bar{q}^{\prime} z}^{\prime}\right)=\left(\begin{array}{c}
\frac{\vec{\sigma} \cdot \vec{p}_{1}^{\prime}}{2 m_{\bar{q}^{\prime}}} \\
G_{1}^{\prime}\left(\vec{p}_{1}^{\prime}\right) \\
G_{1}^{\prime}\left(\vec{p}_{1}^{\prime}\right)
\end{array}\right) \chi_{{\overline{q^{\prime}}}^{\prime} z^{\prime}},
\end{aligned}
$$

where $\vec{\sigma}$ are the Pauli matrices; $\chi_{s_{q z}}, \chi_{s_{\bar{q} z}}, \chi_{s_{q^{\prime} z}}, \chi_{s_{q^{\prime} z}^{\prime}}, \chi_{s_{\bar{q}^{\prime} z}}$, and $\chi_{s_{\bar{q}^{\prime} z}^{\prime}}$ are the spin wave functions with the magnetic projection quantum numbers, $s_{q z}, s_{\bar{q} z}, s_{q^{\prime} z}, s_{q^{\prime} z}^{\prime}, s_{\bar{q}^{\prime} z}$, and $s_{\bar{q}^{\prime} z}^{\prime}$, of the quark or antiquark spin, respectively. The quark and the antiquark created from the gluon have the same mass, i.e., $m_{q}=m_{\bar{q}}$.

Keeping terms to order of the inverse of the quark mass, we get

$\mathcal{M}_{\mathrm{c} q^{\prime} q \bar{q}}=\frac{g_{s}^{2}}{k^{2}} \chi_{s_{q^{\prime} z}^{\prime}}^{+} \chi_{s_{q z}}^{+} T^{e} T^{e} G_{1}^{\prime}\left(\vec{p}_{1}^{\prime}\right) G_{3}\left(\vec{p}_{3}\right)\left[\frac{\vec{\sigma}(34) \cdot \vec{k}}{2 m_{q}}-\frac{\vec{\sigma}(1) \cdot \vec{\sigma}(34) \vec{\sigma}(1) \cdot \vec{p}_{1}+\vec{\sigma}(1) \cdot \vec{p}_{1}^{\prime} \vec{\sigma}(1) \cdot \vec{\sigma}(34)}{2 m_{q^{\prime}}}\right] G_{1}\left(\vec{p}_{1}\right) G_{4}\left(\vec{p}_{4}\right) \chi_{s_{q^{\prime} z}} \chi_{s_{\bar{q} z}}$,

$\mathcal{M}_{\mathrm{c} \bar{q}^{\prime} q \bar{q}}=-\frac{g_{s}^{2}}{k^{2}} \chi_{s_{\bar{q}^{\prime}}}^{+} \chi_{s_{q z}}^{+} T^{e} T^{e} G_{1}\left(\vec{p}_{1}\right) G_{3}\left(\vec{p}_{3}\right)\left[\frac{\vec{\sigma}(34) \cdot \vec{k}}{2 m_{q}}-\frac{\vec{\sigma}(1) \cdot \vec{p}_{1} \vec{\sigma}(1) \cdot \vec{\sigma}(34)+\vec{\sigma}(1) \cdot \vec{\sigma}(34) \vec{\sigma}(1) \cdot \vec{p}_{1}^{\prime}}{2 m_{\bar{q}^{\prime}}}\right] G_{1}^{\prime}\left(\vec{p}_{1}^{\prime}\right) G_{4}\left(\vec{p}_{4}\right) \chi_{s_{\bar{q}^{\prime} z}^{\prime}} \chi_{\bar{q}_{\bar{q} z}}$.

Using $T^{e} T^{e}=\frac{\vec{\lambda}(1)}{2} \cdot \frac{\vec{\lambda}(34)}{2}$ with $\vec{\lambda}$ being the Gell-Mann matrices, we obtain the transition potential for $q^{\prime}\left(p_{1}\right) \rightarrow q^{\prime}\left(p_{1}^{\prime}\right)+q\left(p_{3}\right)+\bar{q}\left(-p_{4}\right)$,

$$
V_{\mathrm{c} q^{\prime} q \bar{q}}(\vec{k})=\frac{\vec{\lambda}(1)}{2} \cdot \frac{\vec{\lambda}(34)}{2} \frac{g_{\mathrm{s}}^{2}}{k^{2}}\left(\frac{\vec{\sigma}(34) \cdot \vec{k}}{2 m_{q}}-\frac{\vec{\sigma}(1) \cdot \vec{\sigma}(34) \vec{\sigma}(1) \cdot \vec{p}_{1}+\vec{\sigma}(1) \cdot \vec{p}_{1}^{\prime} \vec{\sigma}(1) \cdot \vec{\sigma}(34)}{2 m_{q^{\prime}}}\right),
$$

and the transition potential for $\bar{q}^{\prime}\left(-p_{1}\right) \rightarrow \bar{q}^{\prime}\left(-p_{1}^{\prime}\right)+q\left(p_{3}\right)+\bar{q}\left(-p_{4}\right)$,

$$
V_{\mathrm{c} \bar{q}^{\prime} q \bar{q}}(\vec{k})=-\frac{\vec{\lambda}(1)}{2} \cdot \frac{\vec{\lambda}(34)}{2} \frac{g_{\mathrm{s}}^{2}}{k^{2}}\left(\frac{\vec{\sigma}(34) \cdot \vec{k}}{2 m_{q}}-\frac{\vec{\sigma}(1) \cdot \vec{p}_{1} \vec{\sigma}(1) \cdot \vec{\sigma}(34)+\vec{\sigma}(1) \cdot \vec{\sigma}(34) \vec{\sigma}(1) \cdot \vec{p}_{1}^{\prime}}{2 m_{\bar{q}^{\prime}}}\right) .
$$

In Eqs. (51) and (52), $\vec{\lambda}(34)(\vec{\sigma}(34))$ mean that they have matrix elements between the color (spin) wave functions of the final quark and the final antiquark. In Eq. (51), $\vec{\lambda}(1)(\vec{\sigma}(1))$ mean that they have matrix elements between the color (spin) wave functions of the final quark and the initial quark. In Eq. (52), $\vec{\lambda}(1)(\vec{\sigma}(1))$ mean that they have matrix elements between the color (spin) wave functions of the initial antiquark and the final antiquark. Applying Eqs. (51) and (52) to the eight Feynman diagrams, we have $V_{\mathrm{D}_{1}} \equiv V_{\mathrm{c} q_{1} q_{3} \bar{q}_{4}}, V_{\mathrm{D}_{2}} \equiv V_{\mathrm{c}_{1} q_{3} \bar{q}_{4}}, V_{\mathrm{D}_{3}} \equiv V_{\mathrm{c} q_{2} q_{3} \bar{q}_{4}}, V_{\mathrm{D}_{4}} \equiv V_{\bar{c}_{2} q_{3} \bar{q}_{4}}, V_{\mathrm{D}_{5}} \equiv V_{\mathrm{c} q_{1} q_{3} \bar{q}_{4}}$, $V_{\mathrm{D}_{6}} \equiv V_{\mathrm{c} \bar{q}_{1} q_{3} \bar{q}_{4}}, V_{\mathrm{D}_{7}} \equiv V_{\mathrm{c} q_{2} q_{3} \bar{q}_{4}}$, and $V_{\mathrm{D}_{8}} \equiv V_{\mathrm{c} \bar{q}_{2} q_{3} \bar{q}_{4}}$. 


\section{MATRIX ELEMENTS}

The transition amplitudes include color, spin, and flavor matrix elements. Denote the spin of meson $A\left(B, C_{1}, C_{2}\right.$, $\left.C_{3}\right)$ by $S_{A}\left(S_{B}, S_{C_{1}}, S_{C_{2}}, S_{C_{3}}\right)$ and its magnetic projection quantum number by $S_{A z}\left(S_{B z}, S_{C_{1} z}, S_{C_{2} z}, S_{C_{3} z}\right)$. Let $\phi_{A r e l}$ $\left(\phi_{B \text { rel }}, \phi_{C_{1} \text { rel }}, \phi_{C_{2} \text { rel }}, \phi_{C_{3} \text { rel }}\right), \phi_{\text {Acolor }}\left(\phi_{B \text { color }}, \phi_{C_{1} \text { color }}\right.$, $\left.\phi_{C_{2} \text { color }}, \phi_{C_{3} \text { color }}\right), \phi_{\text {Aflavor }}\left(\phi_{B f \text { flavor }}, \phi_{C_{1} \text { flavor }}, \phi_{C_{2} \text { flavor }}\right.$, $\left.\phi_{C_{3} \text { flavor }}\right)$, and $\chi_{S_{A} S_{A z}}\left(\chi_{S_{B} S_{B z}}, \chi_{S_{C_{1}} S_{C_{1} z}}, \chi_{S_{C_{2}} S_{C_{2} z}}, \chi_{S_{C_{3}} S_{C_{3} z}}\right)$ be the quark-antiquark relative-motion wave function, the color wave function, the flavor wave function, and the spin wave function of meson $A\left(B, C_{1}, C_{2}, C_{3}\right)$, respectively. The wave function of mesons $A$ and $B$ is

$\psi_{A B}=\phi_{A \text { rel }} \phi_{B \text { rel }} \phi_{\text {Acolor }} \phi_{B \text { color }} \chi_{S_{A} S_{A z}} \chi_{S_{B} S_{B z}} \varphi_{A B \text { flavor }}$,

and the wave function of mesons $C_{1}, C_{2}$, and $C_{3}$ is

$\psi_{C_{1} C_{2} C_{3}}=\phi_{C_{1} \text { rel }} \phi_{C_{2} \text { rel }} \phi_{C_{3} \text { rel }} \phi_{C_{1} \text { color }} \phi_{C_{2} \text { color }} \phi_{C_{3} \text { color }}$

$$
\times \chi_{S_{C_{1}} S_{C_{1} z}} \chi_{S_{C_{2}} S_{C_{2} z}} \chi_{S_{C_{3}} S_{C_{3} z}} \varphi_{C_{1} C_{2} C_{3} \text { flavor }},
$$

where $\psi_{A B}=\psi_{q_{1} \bar{q}_{1}} \psi_{q_{2} \bar{q}_{2}}$ and $\psi_{C_{1} C_{2} C_{3}}=\psi_{q_{1} \bar{q}_{4}} \psi_{q_{2} \bar{q}_{1}} \psi_{q_{3} \bar{q}_{2}}=$ $\psi_{q_{1} \bar{q}_{2}} \psi_{q_{2} \bar{q}_{4}} \psi_{q_{3} \bar{q}_{1}}$. The flavor wave function $\varphi_{A B f l a v o r}$ of mesons $A$ and $B$ possesses the same isospin $I$ as the flavor

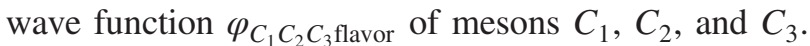

The color wave function of each meson is the color singlet. The color wave function of mesons $A$ and $B$ is $\phi_{A \text { color }} \phi_{B \text { color }}$, and the color wave function of mesons $C_{1}$, $C_{2}$, and $C_{3}$ is $\phi_{C_{1} \text { color }} \phi_{C_{2} \text { color }} \phi_{C_{3} \text { color }}$. The color matrix element is

$$
\phi_{C_{1} \text { color }}^{+} \phi_{C_{2} \text { color }}^{+} \phi_{C_{3} \text { color }}^{+} \frac{\vec{\lambda}}{2} \cdot \frac{\vec{\lambda}(34)}{2} \phi_{\text {Acolor }} \phi_{B \text { color }},
$$

where $\vec{\lambda}$ are the Gell-Mann matrices for the color generators of quark $q_{1}$ in diagram $D_{1}$, antiquark $\bar{q}_{1}$ in diagram $D_{2}$, quark $q_{2}$ in diagram $D_{3}$, antiquark $\bar{q}_{2}$ in diagram $D_{4}$, quark $q_{1}$ in diagram $D_{5}$, antiquark $\bar{q}_{1}$ in diagram $D_{6}$, quark $q_{2}$ in diagram $D_{7}$, or antiquark $\bar{q}_{2}$ in diagram $D_{8}$. The color matrix element is $\frac{4}{9 \sqrt{3}},-\frac{4}{9 \sqrt{3}}, \frac{4}{9 \sqrt{3}},-\frac{4}{9 \sqrt{3}}, \frac{4}{9 \sqrt{3}},-\frac{4}{9 \sqrt{3}}, \frac{4}{9 \sqrt{3}}$, and $-\frac{4}{9 \sqrt{3}}$ for diagrams $D_{1}, D_{2}, D_{3}, D_{4}, D_{5}, D_{6}, D_{7}$, and $D_{8}$, respectively.

The flavor wave functions, $\phi_{\text {Aflavor }}$ and $\phi_{B \text { flavor }}$, are coupled to the flavor wave function $\varphi_{A B \text { flavor }}$. The flavor wave function of meson $C_{1}$ and the one of meson $C_{2}$ are

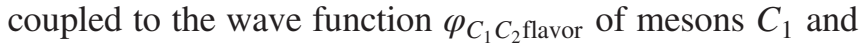
$C_{2}$ with the total isospin $I_{C_{1} C_{2}}^{\mathrm{f}}$. Furthermore, $\varphi_{C_{1} C_{2} \text { flavor }}$ and $\phi_{C_{3} \text { flavor }}$ are coupled to $\varphi_{C_{1} C_{2} C_{3} \text { flavor }}$ with isospin $I$. Let $P_{q_{1} \rightarrow q_{1}+q_{3}+\bar{q}_{4}}\left(P_{\bar{q}_{1} \rightarrow \bar{q}_{1}+q_{3}+\bar{q}_{4}}, P_{q_{2} \rightarrow q_{2}+q_{3}+\bar{q}_{4}}, P_{\bar{q}_{2} \rightarrow \bar{q}_{2}+q_{3}+\bar{q}_{4}}\right)$ denote the operator that implements $q_{1} \rightarrow q_{1}+q_{3}+\bar{q}_{4}$ $\left(\bar{q}_{1} \rightarrow \bar{q}_{1}+q_{3}+\bar{q}_{4}, q_{2} \rightarrow q_{2}+q_{3}+\bar{q}_{4}, \bar{q}_{2} \rightarrow \bar{q}_{2}+q_{3}+\bar{q}_{4}\right)$. The flavor matrix elements corresponding to diagrams $D_{1}$, $D_{2}, D_{3}, D_{4}, D_{5}, D_{6}, D_{7}$, and $D_{8}$ are defined as
TABLE I. Flavor matrix elements in the second column are part of $\mathcal{M}_{\mathrm{D}_{1} \mathrm{f}}, \mathcal{M}_{\mathrm{D}_{2} \mathrm{f}}, \mathcal{M}_{\mathrm{D}_{3} \mathrm{f}}$, and $\mathcal{M}_{\mathrm{D}_{4} \mathrm{f}}$, and in the third column of $\mathcal{M}_{\mathrm{D}_{5} \mathrm{f}}, \mathcal{M}_{\mathrm{D}_{6} \mathrm{f}}, \mathcal{M}_{\mathrm{D}_{7} \mathrm{f}}$, and $\mathcal{M}_{\mathrm{D}_{8} \mathrm{f}}$.

\begin{tabular}{lcc}
$I=2 I_{\pi K}^{\mathrm{f}}=\frac{3}{2} \pi \pi \rightarrow \pi K \bar{K}$ & 1 & 1 \\
$I=1 I_{\pi K}^{\mathrm{f}}=\frac{3}{2} \pi \pi \rightarrow \pi K \bar{K}$ & $\frac{\sqrt{3}}{3}$ & $-\frac{\sqrt{3}}{3}$ \\
$I=1 I_{\pi K}^{\mathrm{f}}=\frac{1}{2} \pi \pi \rightarrow \pi K \bar{K}$ & $\frac{1}{\sqrt{6}}$ & $\frac{1}{\sqrt{6}}$ \\
$I=0 I_{\pi K}^{\mathrm{f}}=\frac{1}{2} \pi \pi \rightarrow \pi K \bar{K}$ & $\frac{1}{2}$ & $-\frac{1}{2}$ \\
$I=\frac{3}{2} I_{\pi K}^{\mathrm{f}}=\frac{3}{2} \pi K \rightarrow \pi \pi K$ & 0 & $\sqrt{\frac{5}{6}}$ \\
$I=\frac{1}{2} I_{\pi K}^{\mathrm{f}}=\frac{3}{2} \pi K \rightarrow \pi \pi K$ & 0 & $\frac{2}{\sqrt{3}}$ \\
$I=\frac{3}{2} I_{\pi K}^{\mathrm{f}}=\frac{1}{2} \pi K \rightarrow \pi \pi K$ & $-\sqrt{\frac{3}{2}}$ & $-\frac{1}{\sqrt{6}}$ \\
$I=\frac{1}{2} I_{\pi K}^{\mathrm{f}}=\frac{1}{2} \pi K \rightarrow \pi \pi K$ & $-\frac{1}{2} \sqrt{\frac{3}{2}}$ & $-\frac{1}{2 \sqrt{6}}$ \\
$I=\frac{3}{2} I_{K K}^{\mathrm{f}}=1 \pi K \rightarrow K K \bar{K}$ & 0 & 1 \\
$I=\frac{1}{2} I_{K K}^{\mathrm{f}}=1 \pi K \rightarrow K K \bar{K}$ & 0 & $-\frac{1}{2}$ \\
$I=\frac{1}{2} I_{K K}^{\mathrm{f}}=0 \pi K \rightarrow K K \bar{K}$ & 0 & $\frac{\sqrt{3}}{2}$ \\
$I=1 I_{\pi K}^{\mathrm{f}}=\frac{3}{2} K K \rightarrow \pi K K$ & $-\frac{2}{\sqrt{3}}$ & $-\frac{2}{\sqrt{3}}$ \\
$I=1 I_{\pi K}^{\mathrm{f}}=\frac{1}{2} K K \rightarrow \pi K K$ & $-\frac{1}{\sqrt{6}}$ & $\frac{1}{\sqrt{6}}$ \\
$I=0 I_{\pi K}^{\mathrm{f}}=\frac{1}{2} K K \rightarrow \pi K K$ & $-\frac{3}{\sqrt{6}}$ & $-\frac{3}{\sqrt{6}}$ \\
$I=1 I_{\pi \bar{K}}^{\mathrm{f}}=\frac{3}{2} K \bar{K} \rightarrow \pi K \bar{K}$ & 0 & $-\frac{2}{\sqrt{3}}$ \\
$I=1 I_{\pi \bar{K}}^{\mathrm{f}}=\frac{1}{2} K \bar{K} \rightarrow \pi K \bar{K}$ & 0 & $\frac{\sqrt{6}}{3}$ \\
$I=0 I_{\pi \bar{K}}^{\mathrm{f}}=\frac{1}{2} K \bar{K} \rightarrow \pi K \bar{K}$ & 0 & 0 \\
\hline \hline
\end{tabular}

$\mathcal{M}_{\mathrm{D}_{1} \mathrm{f}}=\varphi_{C_{1} C_{2} C_{3} \text { flavor }}^{+} P_{q_{1} \rightarrow q_{1}+q_{3}+\bar{q}_{4}} \varphi_{\text {ABflavor }}$,

$\mathcal{M}_{\mathrm{D}_{2} \mathrm{f}}=\varphi_{C_{1} C_{2} C_{3} \text { flavor }}^{+} P_{\bar{q}_{1} \rightarrow \bar{q}_{1}+q_{3}+\bar{q}_{4}} \varphi_{\text {ABflavor }}$,

$\mathcal{M}_{\mathrm{D}_{3} \mathrm{f}}=\varphi_{C_{1} C_{2} C_{3} \text { flavor }}^{+} P_{q_{2} \rightarrow q_{2}+q_{3}+\bar{q}_{4}} \varphi_{\text {ABflavor }}$,

$$
\mathcal{M}_{\mathrm{D}_{4} \mathrm{f}}=\varphi_{C_{1} C_{2} C_{3} \text { flavor }}^{+} P_{\bar{q}_{2} \rightarrow \bar{q}_{2}+q_{3}+\bar{q}_{4}} \varphi_{A B \text { flavor }},
$$

for $A\left(q_{1} \bar{q}_{1}\right)+B\left(q_{2} \bar{q}_{2}\right) \rightarrow C_{1}\left(q_{1} \bar{q}_{4}\right)+C_{2}\left(q_{2} \bar{q}_{1}\right)+C_{3}\left(q_{3} \bar{q}_{2}\right)$ and

$$
\begin{aligned}
& \mathcal{M}_{\mathrm{D}_{5} \mathrm{f}}=\varphi_{C_{1} C_{2} C_{3} \text { flavor }}^{+} P_{q_{1} \rightarrow q_{1}+q_{3}+\bar{q}_{4}} \varphi_{A B \text { flavor }}, \\
& \mathcal{M}_{\mathrm{D}_{6} \mathrm{f}}=\varphi_{C_{1} C_{2} C_{3} \text { flavor }}^{+} P_{\bar{q}_{1} \rightarrow \bar{q}_{1}+q_{3}+\bar{q}_{4}} \varphi_{A B f l a v o r}, \\
& \mathcal{M}_{\mathrm{D}_{7} \mathrm{f}}=\varphi_{C_{1} C_{2} C_{3} \text { flavor }}^{+} P_{q_{2} \rightarrow q_{2}+q_{3}+\bar{q}_{4}} \varphi_{A B f l a v o r}, \\
& \mathcal{M}_{\mathrm{D}_{8} \mathrm{f}}=\varphi_{C_{1} C_{2} C_{3} \text { flavor }}^{+} P_{\bar{q}_{2} \rightarrow \bar{q}_{2}+q_{3}+\bar{q}_{4}} \varphi_{A B \text { flavor }},
\end{aligned}
$$

for $A\left(q_{1} \bar{q}_{1}\right)+B\left(q_{2} \bar{q}_{2}\right) \rightarrow C_{1}\left(q_{1} \bar{q}_{2}\right)+C_{2}\left(q_{2} \bar{q}_{4}\right)+C_{3}\left(q_{3} \bar{q}_{1}\right)$. From the eight Feynman diagrams we have the relation,

$$
\begin{aligned}
& \mathcal{M}_{\mathrm{D}_{1} \mathrm{f}}=\mathcal{M}_{\mathrm{D}_{2} \mathrm{f}}=\mathcal{M}_{\mathrm{D}_{3} \mathrm{f}}=\mathcal{M}_{\mathrm{D}_{4} \mathrm{f}}, \\
& \mathcal{M}_{\mathrm{D}_{5} \mathrm{f}}=\mathcal{M}_{\mathrm{D}_{6} \mathrm{f}}=\mathcal{M}_{\mathrm{D}_{7} \mathrm{f}}=\mathcal{M}_{\mathrm{D}_{8} \mathrm{f}} .
\end{aligned}
$$


TABLE II. Spin matrix elements in $\mathcal{M}_{\mathrm{D}_{1}}, \mathcal{M}_{\mathrm{D}_{2}}, \mathcal{M}_{\mathrm{D}_{3}}, \mathcal{M}_{\mathrm{D}_{4}}, \mathcal{M}_{\mathrm{D}_{5}}, \mathcal{M}_{\mathrm{D}_{6}}, \mathcal{M}_{\mathrm{D}_{7}}$, and $\mathcal{M}_{\mathrm{D}_{8}}$, which are shown from the second to ninth columns, respectively. The initial spin state is $\phi_{\text {iss }}=\chi_{S_{A} S_{A z}} \chi_{S_{B} S_{B z}}$, and the final spin state $\phi_{\mathrm{fss}}=\chi_{S_{C_{1}} S_{C_{1} z}} \chi_{S_{C_{2}} S_{C_{2} z}} \chi_{S_{C_{3}} S_{C_{3} z}}$. The $z$ components of the meson spins are $S_{A z}=S_{B z}=S_{C_{1} z}=S_{C_{2} z}=S_{C_{3} z}=0$.

\begin{tabular}{|c|c|c|c|c|c|c|c|c|}
\hline$\phi_{\mathrm{fss}}^{+} \phi_{\mathrm{iss}}$ & 0 & 0 & 0 & 0 & 0 & 0 & 0 & 0 \\
\hline$\phi_{\mathrm{fss}}^{+} \sigma_{1}(34) \phi_{\text {iss }}$ & 0 & 0 & 0 & 0 & 0 & 0 & 0 & 0 \\
\hline$\phi_{\mathrm{fss}}^{+} \sigma_{2}(34) \phi_{\mathrm{iss}}$ & $-\frac{1}{2 \sqrt{2}} i$ & $-\frac{1}{2 \sqrt{2}} i$ & $-\frac{1}{2 \sqrt{2}} i$ & $-\frac{1}{2 \sqrt{2}} i$ & $-\frac{1}{2 \sqrt{2}} i$ & $-\frac{1}{2 \sqrt{2}} i$ & $-\frac{1}{2 \sqrt{2}} i$ & $-\frac{1}{2 \sqrt{2}} i$ \\
\hline$\phi_{\mathrm{fss}}^{+} \sigma_{3}(34) \phi_{\text {iss }}$ & 0 & 0 & 0 & 0 & 0 & 0 & 0 & 0 \\
\hline$\phi_{\mathrm{fss}}^{+} \sigma_{1} \phi_{\mathrm{iss}}$ & 0 & 0 & 0 & 0 & 0 & 0 & 0 & 0 \\
\hline$\phi_{\mathrm{fss}}^{+} \sigma_{2} \phi_{\mathrm{iss}}$ & $-\frac{1}{2 \sqrt{2}} i$ & $-\frac{1}{2 \sqrt{2}} i$ & $-\frac{1}{2 \sqrt{2}} i$ & $-\frac{1}{2 \sqrt{2}} i$ & $-\frac{1}{2 \sqrt{2}} i$ & $-\frac{1}{2 \sqrt{2}} i$ & $-\frac{1}{2 \sqrt{2}} i$ & $-\frac{1}{2 \sqrt{2}} i$ \\
\hline$\phi_{\mathrm{fss}}^{+} \sigma_{3} \phi_{\mathrm{iss}}$ & 0 & 0 & 0 & 0 & 0 & 0 & 0 & 0 \\
\hline$\phi_{\mathrm{fss}}^{+} \sigma_{1}(34) \sigma_{1} \phi_{\text {iss }}$ & 0 & 0 & 0 & 0 & 0 & 0 & 0 & 0 \\
\hline$\phi_{\mathrm{fss}}^{+} \sigma_{1}(34) \sigma_{2} \phi_{\mathrm{iss}}$ & 0 & 0 & 0 & 0 & 0 & 0 & 0 & 0 \\
\hline$\phi_{\mathrm{fss}}^{+} \sigma_{1}(34) \sigma_{3} \phi_{\mathrm{iss}}$ & $\frac{1}{2 \sqrt{2}}$ & $-\frac{1}{2 \sqrt{2}}$ & $\frac{1}{2 \sqrt{2}}$ & $-\frac{1}{2 \sqrt{2}}$ & $\frac{1}{2 \sqrt{2}}$ & $-\frac{1}{2 \sqrt{2}}$ & $\frac{1}{2 \sqrt{2}}$ & $-\frac{1}{2 \sqrt{2}}$ \\
\hline$\phi_{\mathrm{fss}}^{+} \sigma_{2}(34) \sigma_{1} \phi_{\mathrm{iss}}$ & 0 & 0 & 0 & 0 & 0 & 0 & 0 & 0 \\
\hline$\phi_{\mathrm{fss}}^{+} \sigma_{2}(34) \sigma_{2} \phi_{\text {iss }}$ & 0 & 0 & 0 & 0 & 0 & 0 & 0 & 0 \\
\hline$\phi_{\mathrm{fss}}^{+} \sigma_{2}(34) \sigma_{3} \phi_{\mathrm{iss}}$ & 0 & 0 & 0 & 0 & 0 & 0 & 0 & 0 \\
\hline$\phi_{\mathrm{fss}}^{+} \sigma_{3}(34) \sigma_{1} \phi_{\text {iss }}$ & $-\frac{1}{2 \sqrt{2}}$ & $\frac{1}{2 \sqrt{2}}$ & $-\frac{1}{2 \sqrt{2}}$ & $\frac{1}{2 \sqrt{2}}$ & $-\frac{1}{2 \sqrt{2}}$ & $\frac{1}{2 \sqrt{2}}$ & $-\frac{1}{2 \sqrt{2}}$ & $\frac{1}{2 \sqrt{2}}$ \\
\hline$\phi_{\mathrm{fss}}^{+} \sigma_{3}(34) \sigma_{2} \phi_{\mathrm{iss}}$ & 0 & 0 & 0 & 0 & 0 & 0 & 0 & 0 \\
\hline$\phi_{\mathrm{fss}}^{+} \sigma_{3}(34) \sigma_{3} \phi_{\mathrm{iss}}$ & 0 & 0 & 0 & 0 & 0 & 0 & 0 & 0 \\
\hline
\end{tabular}

We list in Table I the flavor matrix elements for the following 2-to-3 meson-meson reactions:

$$
\begin{array}{rlrl}
\pi \pi & \rightarrow \pi K \bar{K}, & \pi K \rightarrow \pi \pi K, \quad \pi K \rightarrow K K \bar{K}, \\
K K & \rightarrow \pi K K, & K \bar{K} \rightarrow \pi K \bar{K}, &
\end{array}
$$

where $K=\left(\begin{array}{c}K^{+} \\ K^{0}\end{array}\right)$ and $\bar{K}=\left(\begin{array}{c}\bar{K}^{0} \\ K^{-}\end{array}\right)$.

The initial mesons and the final mesons in the five reactions are pseudoscalar mesons. The spin wave function of each pseudoscalar meson is the spin singlet of the quark and the antiquark. The spin wave function of the two initial mesons or the three final mesons is simply the product of the spin wave function of each meson as seen in Eqs. (53) or (54). Spin matrix elements are listed in Table II. In the table $\vec{\sigma}$ are the Pauli matrices for quark $q_{1}$ in diagrams $\mathrm{D}_{1}$ and $\mathrm{D}_{5}$, antiquark $\bar{q}_{1}$ in diagrams $\mathrm{D}_{2}$ and $\mathrm{D}_{6}$, quark $q_{2}$ in diagrams $\mathrm{D}_{3}$ and $\mathrm{D}_{7}$, or antiquark $\bar{q}_{2}$ in diagrams $\mathrm{D}_{4}$ and $\mathrm{D}_{8}$.

The mesonic quark-antiquark relative-motion wave functions, $\phi_{\text {Arel }}, \phi_{B \text { rel }}, \phi_{C_{1} \text { rel }}, \phi_{C_{2} \text { rel }}$, and $\phi_{C_{3} \text { rel }}$, are the solutions of the Schrödinger equation with the potential [61] between constituents $a$ and $b$ :

$$
\begin{aligned}
V_{a b}(\vec{r})= & -\frac{\vec{\lambda}_{a}}{2} \cdot \frac{\vec{\lambda}_{b}}{2} \frac{3}{4} D\left[1.3-\left(\frac{T}{T_{\mathrm{c}}}\right)^{4}\right] \tanh (A r)+\frac{\vec{\lambda}_{a}}{2} \cdot \frac{\vec{\lambda}_{b}}{2} \frac{6 \pi}{25} \frac{v(\lambda r)}{r} \exp (-E r) \\
& -\frac{\vec{\lambda}_{a}}{2} \cdot \frac{\vec{\lambda}_{b}}{2} \frac{16 \pi^{2}}{25} \frac{d^{3}}{\pi^{3 / 2}} \exp \left(-d^{2} r^{2}\right) \frac{\vec{s}_{a} \cdot \vec{s}_{b}}{m_{a} m_{b}}+\frac{\vec{\lambda}_{a}}{2} \cdot \frac{\vec{\lambda}_{b}}{2} \frac{4 \pi}{25} \frac{1}{r} \frac{d^{2} v(\lambda r)}{d r^{2}} \frac{\vec{s}_{a} \cdot \vec{s}_{b}}{m_{a} m_{b}}
\end{aligned}
$$

in which $D=0.7 \mathrm{GeV}, E=0.6 \mathrm{GeV}, \lambda=\sqrt{25 / 16 \pi^{2} \alpha^{\prime}}$ with $\alpha^{\prime}=1.04 \mathrm{GeV}^{-2}$, and $A=1.5\left[0.75+0.25\left(T / T_{\mathrm{c}}\right)^{10}\right]^{6} \mathrm{GeV}$, where $T$ is the temperature and $T_{\mathrm{c}}$ is the critical temperature which equals $0.175 \mathrm{GeV}$ [62]. The function $v$ is given by Buchmüller and Tye in Ref. [63], and the quantity $d$ is given by

$d^{2}=d_{1}^{2}\left[\frac{1}{2}+\frac{1}{2}\left(\frac{4 m_{a} m_{b}}{\left(m_{a}+m_{b}\right)^{2}}\right)^{4}\right]+d_{2}^{2}\left(\frac{2 m_{a} m_{b}}{m_{a}+m_{b}}\right)^{2}$, where $d_{1}=0.15 \mathrm{GeV}$ and $d_{2}=0.705$. The potential is a function of the distance $r$ between constituents $a$ and $b$, and contains the spins, $\vec{s}_{a}$ and $\vec{s}_{b}$, and the Gell-Mann matrices $\vec{\lambda}_{a}$ and $\vec{\lambda}_{b}$. When the masses of the up quark, the down quark, the strange quark, and the charm quark are $0.32 \mathrm{GeV}, 0.32 \mathrm{GeV}, 0.5 \mathrm{GeV}$, and $1.51 \mathrm{GeV}$, respectively, the meson masses obtained from the Schrödinger equation with the potential at zero temperature reproduce the experimental masses of $\pi, \rho, K, K^{*}, J / \psi, \psi^{\prime}, \chi_{c}, D, D^{*}$, 


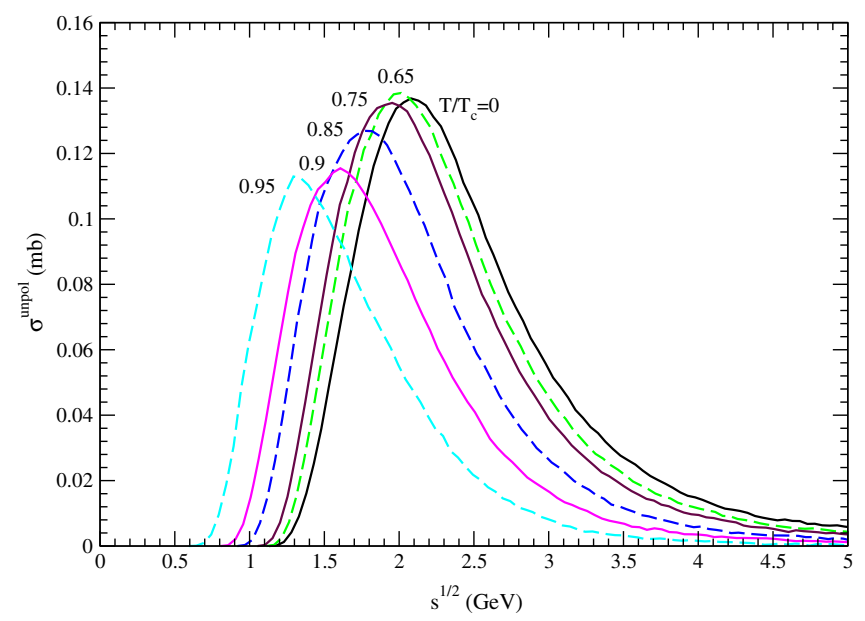

FIG. 4. Cross sections for $\pi \pi \rightarrow \pi K \bar{K}$ for $I=2$ and $I_{\pi K}^{f}=3 / 2$ at various temperatures (in units of the critical temperature).

$D_{s}$, and $D_{s}^{*}$ mesons [64]. Moreover, the experimental data of $S$-wave phase shifts for elastic $\pi \pi$ scattering for $I=2$ in vacuum $[2,3,6,10]$ are reproduced in the Born approximation.

\section{NUMERICAL CROSS SECTIONS AND DISCUSSIONS}

We consider the following 2-to-3 meson-meson reactions:

$$
\begin{aligned}
\pi \pi & \rightarrow \pi K \bar{K}, & \pi K \rightarrow \pi \pi K, & \pi K \rightarrow K K \bar{K}, \\
K K & \rightarrow \pi K K, & K \bar{K} & \rightarrow \pi K \bar{K} .
\end{aligned}
$$

The reaction $\pi \bar{K} \rightarrow \pi \pi \bar{K}(\pi \bar{K} \rightarrow K \bar{K} \bar{K}, \bar{K} \bar{K} \rightarrow \pi \bar{K} \bar{K})$ has the same cross section as $\pi K \rightarrow \pi \pi K(\pi K \rightarrow K K \bar{K}$, $K K \rightarrow \pi K K)$. Cross sections for meson-meson reactions depend on the flavor matrix elements. Based on the flavor matrix elements, cross sections for some isospin channels of reactions can be obtained from the other isospin channels. Therefore, we calculate cross sections for the following eight channels:

$$
\begin{aligned}
& I=2 I_{\pi K}^{\mathrm{f}}=\frac{3}{2} \pi \pi \rightarrow \pi K \bar{K}, \quad I=1 I_{\pi K}^{\mathrm{f}}=\frac{3}{2} \pi \pi \rightarrow \pi K \bar{K}, \\
& I=\frac{3}{2} I_{\pi K}^{\mathrm{f}}=\frac{3}{2} \pi K \rightarrow \pi \pi K, \quad I=\frac{3}{2} I_{\pi K}^{\mathrm{f}}=\frac{1}{2} \pi K \rightarrow \pi \pi K, \\
& I=\frac{3}{2} I_{K K}^{\mathrm{f}}=1 \pi K \rightarrow K K \bar{K}, \quad I=1 I_{\pi K}^{\mathrm{f}}=\frac{3}{2} K K \rightarrow \pi K K, \\
& I=1 I_{\pi K}^{\mathrm{f}}=\frac{1}{2} K K \rightarrow \pi K K, \quad I=1 I_{\pi \bar{K}}^{\mathrm{f}}=\frac{3}{2} K \bar{K} \rightarrow \pi K \bar{K} .
\end{aligned}
$$

According to Eq. (40) we calculate the unpolarized cross section at the six temperatures $T / T_{\mathrm{c}}=0,0.65,0.75,0.85$, 0.9 , and 0.95 . We plot the unpolarized cross sections for the eight channels of the reactions in Figs. 4-11. These cross

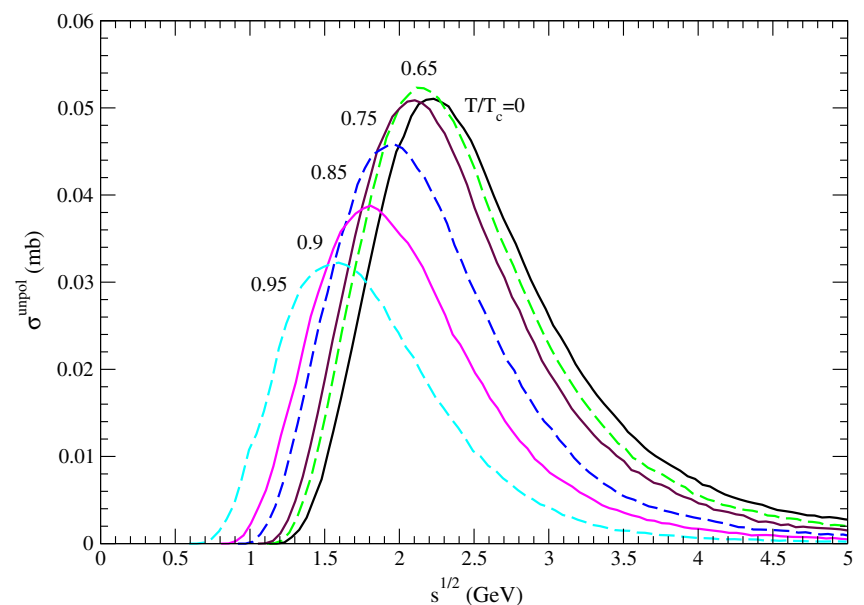

FIG. 5. Cross sections for $\pi \pi \rightarrow \pi K \bar{K}$ for $I=1$ and $I_{\pi K}^{f}=3 / 2$ at various temperatures.

sections are functions of the temperature of hadronic matter and the Mandelstam variable $\sqrt{s}$.

Every curve in Figs. $4-11$ has a peak. Let $\sqrt{s_{0}}$ be the threshold energy. Denote by $d_{0}$ the separation between the peak's location on the $\sqrt{s}$-axis and the threshold energy. The numerical cross sections shown in Figs. 4-11 are parametrized as

$$
\begin{aligned}
\sigma^{\text {unpol }}(\sqrt{s}, T)= & a_{1}\left(\frac{\sqrt{s}-\sqrt{s_{0}}}{b_{1}}\right)^{e_{1}} \exp \left[e_{1}\left(1-\frac{\sqrt{s}-\sqrt{s_{0}}}{b_{1}}\right)\right] \\
& +a_{2}\left(\frac{\sqrt{s}-\sqrt{s_{0}}}{b_{2}}\right)^{e_{2}} \exp \left[e_{2}\left(1-\frac{\sqrt{s}-\sqrt{s_{0}}}{b_{2}}\right)\right] .
\end{aligned}
$$

The values of the parameters $a_{1}, b_{1}, e_{1}, a_{2}, b_{2}$, and $e_{2}$ are listed in Tables III and IV, where $\sqrt{s_{\mathrm{Z}}}$ is the square root of the Mandelstam variable at which the cross section is $1 / 100$ of the peak cross section.

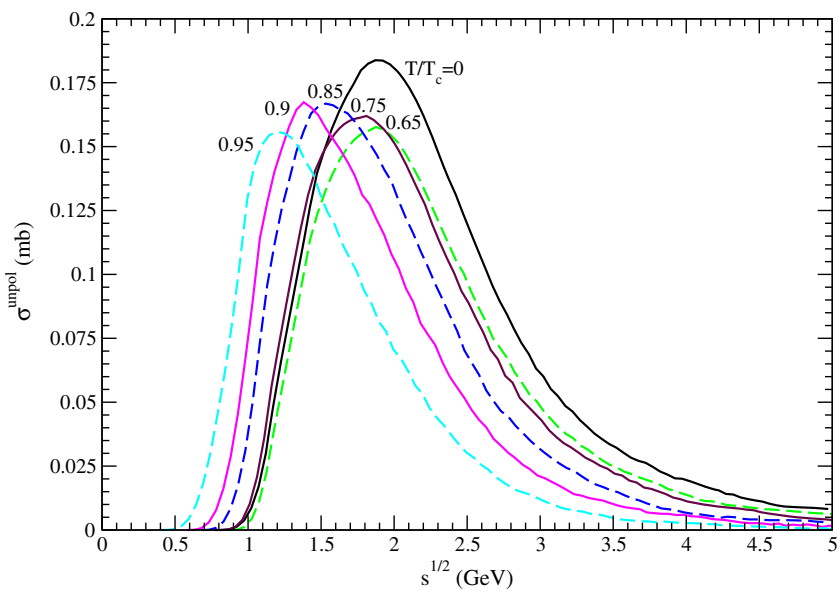

FIG. 6. Cross sections for $\pi K \rightarrow \pi \pi K$ for $I=3 / 2$ and $I_{\pi K}^{f}=$ $3 / 2$ at various temperatures. 


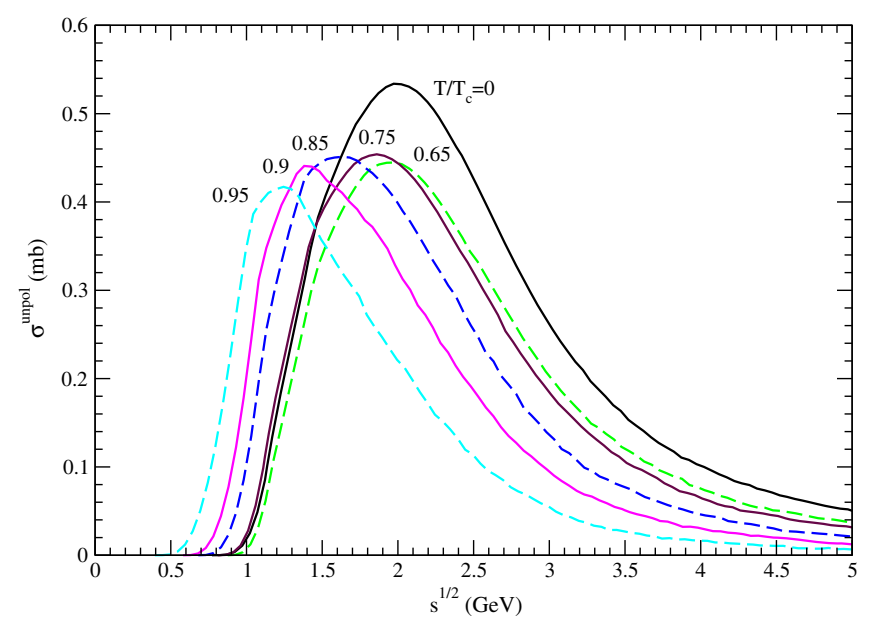

FIG. 7. Cross sections for $\pi K \rightarrow \pi \pi K$ for $I=3 / 2$ and $I_{\pi K}^{f}=$ $1 / 2$ at various temperatures.

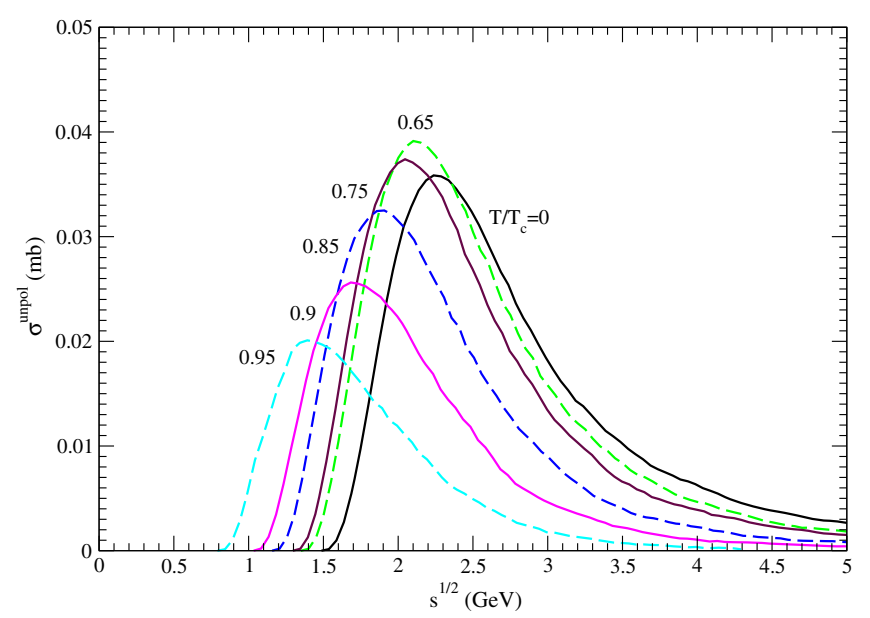

FIG. 8. Cross sections for $\pi K \rightarrow K K \bar{K}$ for $I=3 / 2$ and $I_{K K}^{f}=$ 1 at various temperatures.

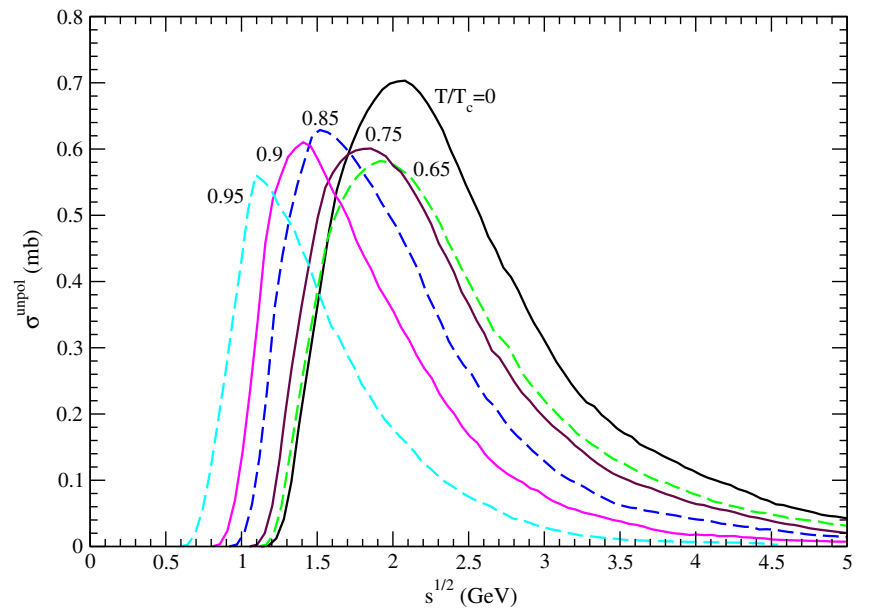

FIG. 9. Cross sections for $K K \rightarrow \pi K K$ for $I=1$ and $I_{\pi K}^{f}=$ $3 / 2$ at various temperatures.

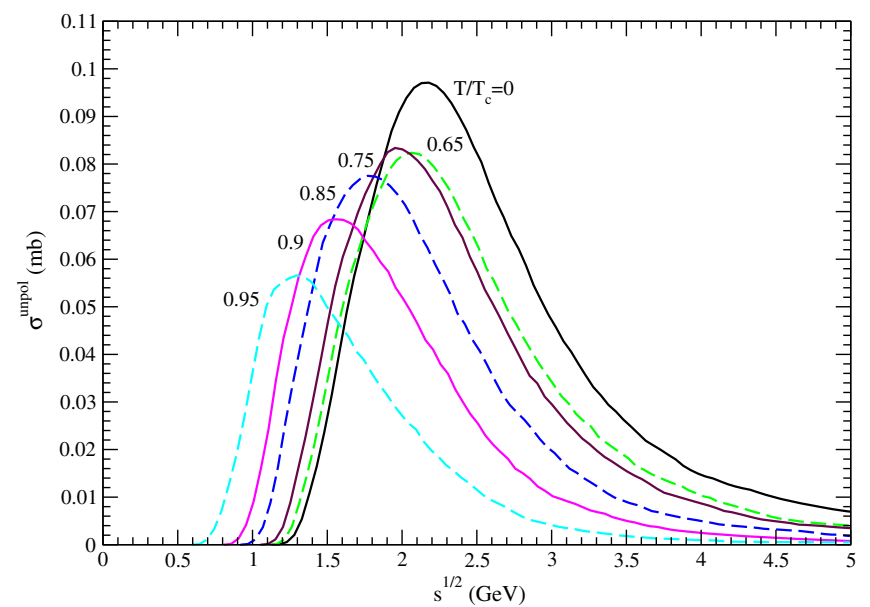

FIG. 10. Cross sections for $K K \rightarrow \pi K K$ for $I=1$ and $I_{\pi K}^{f}=$ $1 / 2$ at various temperatures.

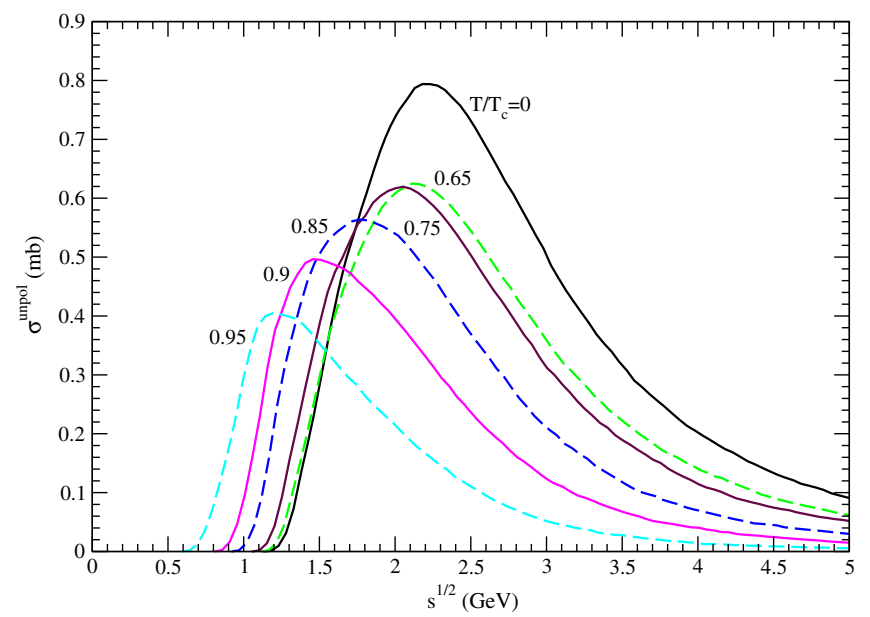

FIG. 11. Cross sections for $K \bar{K} \rightarrow \pi K \bar{K}$ for $I=1$ and $I_{\pi \bar{K}}^{f}=$ $3 / 2$ at various temperatures.

Since the sum of the masses of the final mesons is larger than the sum of the masses of the initial mesons, the reactions are endothermic. When $\sqrt{s}$ increases from the threshold energy, which is the sum of the masses of the final mesons, the cross section of every reaction shown in Figs. 4-11 increases from zero to a maximum and then decreases. The change of the peak cross section with temperature is obvious, and the peak cross section at $T / T_{\mathrm{c}}=0.95$ is smallest among the peak cross sections at the six temperatures.

As the temperature increases, values of the central spinindependent potential [the first term and the second term of the right-hand side in Eq. (65)] at large distances become smaller and smaller (confinement becomes weaker and weaker), and the Schrödinger equation produces increasing meson radii. The weakening confinement with increasing temperature makes combining final quarks and antiquarks 
TABLE III. Values of the parameters. $a_{1}$ and $a_{2}$ are in units of millibarns; $b_{1}, b_{2}, d_{0}$, and $\sqrt{s_{\mathrm{Z}}}$ are in units of GeV; $e_{1}$ and $e_{2}$ are dimensionless.

\begin{tabular}{llllllllll}
\hline \hline Reactions & $T / T_{\mathrm{c}}$ & $a_{1}$ & $b_{1}$ & $e_{1}$ & $a_{2}$ & $b_{2}$ & $e_{2}$ & $d_{0}$ & $\sqrt{s_{\mathrm{z}}}$ \\
\hline$I=2 I_{\pi K}^{\mathrm{f}}=\frac{3}{2} \pi \pi \rightarrow \pi K \bar{K}$ & 0 & 0.1 & 0.81 & 3.81 & 0.05 & 1.4 & 5.06 & 0.95 & 5.83 \\
& 0.65 & 0.09 & 0.72 & 3.24 & 0.06 & 1.19 & 4.12 & 0.9 & 5.82 \\
& 0.75 & 0.09 & 0.71 & 3.37 & 0.06 & 1.18 & 4.16 & 0.9 & 5.6 \\
& 0.85 & 0.08 & 0.64 & 3.69 & 0.07 & 1.13 & 4.59 & 0.85 & 5.06 \\
& 0.9 & 0.07 & 0.62 & 3.57 & 0.06 & 1.07 & 4.31 & 0.8 & 5.01 \\
$I=1 I_{\pi K}^{\mathrm{f}}=\frac{3}{2} \pi \pi \rightarrow \pi K \bar{K}$ & 0.95 & 0.07 & 0.6 & 4.95 & 0.06 & 1.05 & 4.68 & 0.7 & 4.26 \\
& 0 & 0.03 & 1.04 & 4.88 & 0.02 & 1.27 & 2.9 & 1.1 & 6.08 \\
& 0.65 & 0.03 & 1.01 & 3.55 & 0.02 & 1.09 & 3.14 & 1.05 & 5.52 \\
& 0.75 & 0.03 & 0.99 & 4.5 & 0.02 & 1.11 & 2.64 & 1.05 & 5.37 \\
& 0.85 & 0.03 & 0.84 & 3.8 & 0.02 & 1.33 & 5.97 & 1.05 & 5.15 \\
& 0.9 & 0.02 & 0.81 & 3.5 & 0.02 & 1.19 & 5.06 & 1 & 5.08 \\
& 0.95 & 0.02 & 0.91 & 3.79 & 0.01 & 1.09 & 3.87 & 1 & 4.63 \\
& 0 & 0.13 & 0.81 & 5.5 & 0.11 & 1.52 & 6.67 & 1.1 & 6.36 \\
$\frac{3}{2} I_{\pi K}^{\mathrm{f}}=\frac{3}{2} \pi K \rightarrow \pi \pi K$ & 0.65 & 0.1 & 0.67 & 4.29 & 0.1 & 1.31 & 5.2 & 1 & 5.87 \\
& 0.75 & 0.1 & 0.69 & 4.51 & 0.1 & 1.24 & 4.3 & 1 & 5.35 \\
& 0.85 & 0.12 & 0.68 & 5.27 & 0.1 & 1.28 & 5.72 & 0.85 & 5.15 \\
& 0.9 & 0.11 & 0.68 & 5.36 & 0.1 & 1.19 & 5.56 & 0.8 & 4.89 \\
& 0.95 & 0.12 & 0.78 & 5.66 & 0.06 & 1.26 & 5 & 0.8 & 4.38 \\
& 0 & 0.35 & 0.84 & 5.17 & 0.34 & 1.66 & 4.84 & 1.2 & 5.8 \\
$\frac{3}{2} I_{\pi K}^{\mathrm{f}}=\frac{1}{2} \pi K \rightarrow \pi \pi K$ & 0.65 & 0.29 & 0.73 & 3.94 & 0.27 & 1.5 & 4.18 & 1.1 & 6.62 \\
& 0.75 & 0.3 & 0.7 & 4.62 & 0.3 & 1.44 & 4.27 & 1.05 & 5.97 \\
& 0.85 & 0.3 & 0.69 & 5.04 & 0.3 & 1.36 & 4.49 & 0.95 & 5.82 \\
& 0.9 & 0.29 & 0.67 & 5.51 & 0.29 & 1.3 & 4.81 & 0.85 & 5.49 \\
& 0.95 & 0.27 & 0.77 & 6.41 & 0.21 & 1.23 & 3.59 & 0.85 & 5.24 \\
\hline \hline
\end{tabular}

TABLE IV. The same as Table III, but for four other reactions.

\begin{tabular}{|c|c|c|c|c|c|c|c|c|c|}
\hline Reactions & $T / T_{\mathrm{c}}$ & $a_{1}$ & $b_{1}$ & $e_{1}$ & $a_{2}$ & $b_{2}$ & $e_{2}$ & $d_{0}$ & $\sqrt{s_{\mathrm{z}}}$ \\
\hline \multirow{6}{*}{$I=\frac{3}{2} I_{K K}^{\mathrm{f}}=1 \pi K \rightarrow K K \bar{K}$} & 0 & 0.02 & 0.6 & 3.92 & 0.02 & 1.08 & 2.82 & 0.75 & 6 \\
\hline & 0.65 & 0.02 & 0.68 & 3.75 & 0.02 & 0.95 & 2.15 & 0.75 & 5.85 \\
\hline & 0.75 & 0.03 & 0.69 & 3.01 & 0.01 & 1.19 & 2.69 & 0.75 & 5.64 \\
\hline & 0.85 & 0.02 & 0.52 & 3.65 & 0.02 & 1.03 & 4.07 & 0.75 & 5.6 \\
\hline & 0.9 & 0.01 & 0.43 & 3.19 & 0.02 & 0.87 & 3.57 & 0.65 & 5.49 \\
\hline & 0.95 & 0.01 & 0.6 & 3.22 & 0.01 & 0.75 & 1.87 & 0.6 & 4.26 \\
\hline \multirow{6}{*}{$I=1 I_{\pi K}^{\mathrm{f}}=\frac{3}{2} K K \rightarrow \pi K K$} & 0 & 0.46 & 0.65 & 3.43 & 0.38 & 1.26 & 3.11 & 0.95 & 6.15 \\
\hline & 0.65 & 0.38 & 0.64 & 2.63 & 0.27 & 1.16 & 2.52 & 0.8 & 5.91 \\
\hline & 0.75 & 0.4 & 0.55 & 3.08 & 0.35 & 1.14 & 3.47 & 0.8 & 5.54 \\
\hline & 0.85 & 0.41 & 0.47 & 4.2 & 0.41 & 1.03 & 3.81 & 0.6 & 5.52 \\
\hline & 0.9 & 0.4 & 0.45 & 4.69 & 0.39 & 0.94 & 3.93 & 0.6 & 5.49 \\
\hline & 0.95 & 0.38 & 0.51 & 4.4 & 0.25 & 0.9 & 3.67 & 0.5 & 4.3 \\
\hline \multirow{6}{*}{$I=1 I_{\pi K}^{\mathrm{f}}=\frac{1}{2} K K \rightarrow \pi K K$} & 0 & 0.07 & 0.92 & 3.64 & 0.03 & 1.44 & 3.16 & 1.05 & 6.49 \\
\hline & 0.65 & 0.07 & 0.83 & 3.13 & 0.02 & 1.56 & 4.55 & 0.95 & 6.72 \\
\hline & 0.75 & 0.05 & 0.73 & 3.22 & 0.04 & 1.21 & 3.54 & 0.9 & 6.27 \\
\hline & 0.85 & 0.05 & 0.67 & 3.63 & 0.04 & 1.15 & 3.72 & 0.85 & 5.52 \\
\hline & 0.9 & 0.04 & 0.54 & 4.52 & 0.05 & 1.04 & 4.58 & 0.75 & 5.08 \\
\hline & 0.95 & 0.04 & 0.58 & 4.41 & 0.03 & 1.08 & 5.14 & 0.7 & 4.53 \\
\hline \multirow{6}{*}{$I=1 I_{\pi \bar{K}}^{\mathrm{f}}=\frac{3}{2} K \bar{K} \rightarrow \pi K \bar{K}$} & 0 & 0.52 & 0.83 & 3.01 & 0.38 & 1.61 & 3.38 & 1.05 & 6.3 \\
\hline & 0.65 & 0.44 & 0.84 & 2.52 & 0.23 & 1.51 & 2.54 & 1 & 6.18 \\
\hline & 0.75 & 0.42 & 0.73 & 2.72 & 0.3 & 1.45 & 3.27 & 1 & 6.05 \\
\hline & 0.85 & 0.37 & 0.57 & 3.56 & 0.38 & 1.26 & 3.7 & 0.85 & 5.66 \\
\hline & 0.9 & 0.34 & 0.53 & 3.81 & 0.32 & 1.16 & 3.7 & 0.65 & 5.64 \\
\hline & 0.95 & 0.28 & 0.56 & 4.01 & 0.22 & 1.09 & 3.68 & 0.6 & 5.22 \\
\hline
\end{tabular}


into mesons more difficult, and thus reduces the cross sections. In contrast to decreasing peak cross sections caused by weakening confinement, increasing peak cross sections are caused by increasing radii of the initial mesons. When the decrease is faster than the increase, the peak cross section goes down as the temperature changes from a value (for example, $0.65 T_{\mathrm{c}}$ in Fig. 8 that show cross sections for $\pi K \rightarrow K K \bar{K}$ for $I=3 / 2$ and $\left.I_{K K}^{f}=1\right)$ to $0.95 T_{\mathrm{c}}$.

With increasing temperature, the meson radii increase. This corresponds to increasing wave functions at small quark-antiquark relative momentum. The relative momentum depends linearly on the three-dimensional momentum $\vec{P}$ of an initial meson in the center-of-mass frame of the two initial mesons,

$$
\vec{P}^{2}=\frac{1}{4}\left[s-\left(m_{A}+m_{B}\right)^{2}\right]\left[1-\frac{\left(m_{A}-m_{B}\right)^{2}}{s}\right] .
$$

The small relative momentum may be given by small values of $|\vec{P}|$ and, furthermore, of $\sqrt{s}$. A consequence is that $d_{0}$ listed in Tables III and IV decreases or stays unchanged with increasing temperature. The peak cross section occurs at $\sqrt{s}=m_{C_{1}}+m_{C_{2}}+m_{C_{3}}+d_{0}$. With increasing temperature, the decrease of the pion and kaon masses [52] in addition to $d_{0}$ lead to the decrease of $\sqrt{s}=m_{C_{1}}+m_{C_{2}}+m_{C_{3}}+d_{0}$.

Cross sections for $\pi K \rightarrow \pi \pi K$ for $I=3 / 2$ were measured in Ref. [27], but systematic and statistical uncertainties were not given. The experimental cross section is $0.04 \mathrm{mb}$ at $\sqrt{s}=0.95 \mathrm{GeV}$ and $0.16 \mathrm{mb}$ at $\sqrt{s}=1.15 \mathrm{GeV}$. The two data are individually near the values $0.014 \mathrm{mb}$ and $0.2 \mathrm{mb}$ of the present work at zero temperature.

The 2-to-3 meson-meson scattering is caused by a gluon created from a quark or an antiquark and the gluon creates a quark-antiquark pair. If the quark-antiquark pair is $u \bar{u}$ or $d \bar{d}$, we have the reaction $\pi K \rightarrow \pi \pi K$. If the quark-antiquark pair is $s \bar{s}$, we have the reaction $\pi K \rightarrow K K \bar{K}$. Since the upquark and down-quark masses are smaller than the strangequark mass, it is more likely to create a $u \bar{u}$ or $d \bar{d}$ pair than a $s \bar{s}$ pair. Therefore, the peak cross sections of $\pi K \rightarrow \pi \pi K$ for $I=3 / 2$ at a given temperature in Figs. 6 and 7 are larger than the one of $\pi K \rightarrow K K \bar{K}$ for $I=3 / 2$ and $I_{K K}^{\mathrm{f}}=$ 1 in Fig. 8.

Some 2-to-3 meson-meson reactions in the present work and some 2-to-2 meson-meson reactions in Ref. [53] have the same initial mesons. We can thus compare the cross sections obtained in the present work and those provided in Ref. [53]. At a given temperature the peak cross section of $\pi \pi \rightarrow \pi K \bar{K}$ for $I=1$ and $I_{\pi K}^{\mathrm{f}}=3 / 2$ in Fig. 5 is smaller than the one of $\pi \pi \rightarrow K \bar{K}^{*}$ for $I=1$ in Ref. [53]. Cross sections for $\pi K \rightarrow \pi \pi K$ for $I=3 / 2$ are shown in Figs. 6 and 7. According to the flavor matrix elements in Table I, the cross section for $\pi K \rightarrow \pi \pi K$ for $I=1 / 2$ and
$I_{\pi K}^{\mathrm{f}}=3 / 2$ is 1.6 times the one for $\pi K \rightarrow \pi \pi K$ for $I=3 / 2$ and $I_{\pi K}^{\mathrm{f}}=3 / 2$, and the cross section for $\pi K \rightarrow \pi \pi K$ for $I=1 / 2$ and $I_{\pi K}^{\mathrm{f}}=1 / 2$ is 0.25 times the one for $\pi K \rightarrow$ $\pi \pi K$ for $I=3 / 2$ and $I_{\pi K}^{\mathrm{f}}=1 / 2$. The peak cross section of $\pi K \rightarrow \pi \pi K$ for $I=1 / 2$ and $I_{\pi K}^{\mathrm{f}}=3 / 2$ is smaller than the one of $\pi K \rightarrow \pi K^{*}$ for $I=1 / 2$ at $T / T_{\mathrm{c}}=0,0.65$, and 0.75 , but larger at $T / T_{\mathrm{c}}=0.85,0.9$, and 0.95 . The peak cross section of $\pi K \rightarrow \pi \pi K$ for $I=1 / 2$ and $I_{\pi K}^{\mathrm{f}}=1 / 2$ is smaller than the one of $\pi K \rightarrow \pi K^{*}$ for $I=1 / 2$. The peak cross section of $K \bar{K} \rightarrow \pi K \bar{K}$ for $I=1$ and $I_{\pi \bar{K}}^{\mathrm{f}}=3 / 2$ in Fig. 11 is smaller than the one of $K \bar{K} \rightarrow K \bar{K}^{*}$ for $I=1$ at $T / T_{\mathrm{c}}=0,0.65,0.75$, and 0.95 , but larger at $T / T_{\mathrm{c}}=$ 0.85 and 0.9. Therefore, 2-to-3 meson-meson scattering may be as important as inelastic 2-to-2 meson-meson scattering.

The $\tau$ decay has been used to study asymptotic freedom of QCD $[65,66]$. The $\tau$ lepton decays into $\nu_{\tau}$ and $W$ which splits into a quark and an antiquark. If the quark or the antiquark emits a virtual gluon which subsequently splits into a quark-antiquark pair, decay modes like $\tau^{-} \rightarrow \pi^{-} \bar{K}^{0} \nu_{\tau}$ and $\tau^{-} \rightarrow K^{-} K^{0} \nu_{\tau}$ are observed. If the quark and/or the antiquark creates two virtual gluons of which each subsequently splits into a quark-antiquark pair, decay modes like $\tau^{-} \rightarrow \pi^{-} \bar{K}^{0} \pi^{0} \nu_{\tau}$ and $\tau^{-} \rightarrow K^{-} K^{0} \pi^{0} \nu_{\tau}$ are observed. That the virtual gluon splits into a quark-antiquark pair also takes place in 2-to-3 meson-meson scattering in the present work, and perturbative QCD is applied to the process.

In perturbative QCD physical observables are usually given by a power series in $\alpha_{\mathrm{s}}$, which is $g_{s}^{2} / 4 \pi$. If the coupling constant $\alpha_{\mathrm{s}}$ is smaller than 1, the perturbative expansion converges. In the present work the coupling constant is 0.75 from Ref. [63], and is used in the Feynman diagrams shown in Fig. 3.

When we add the gluon propagator, the gluon loop, the quark loop, and the ghost loop to the eight diagrams in Figs. 1 and 2, this generates 312 Feynman diagrams at order $\alpha_{\mathrm{s}}^{2}$. The 312 diagrams and the 8 diagrams in Figs. 1 and 2 do not contain quark-antiquark annihilation. From the annihilation of an initial quark and an initial antiquark as well as the creation of a quark-antiquark pair, we get 14 Feynman diagrams at order $\alpha_{\mathrm{s}}^{2}$. In total, we have 326 diagrams. The calculation of such a large number of diagrams is formidable.

The transition potentials given in Eqs. (51) and (52) consist of terms with the inverse of the quark mass. In obtaining the transition potentials the terms with the inverse of the quark mass cubed are neglected, because they are suppressed by the inverse of the quark mass squared in comparison to the terms in Eqs. (51) and (52).

Nonperturbative effects exist in 2-to-3 meson-meson scattering, and are encoded in the mesonic quark-antiquark wave functions as done in Refs. [67,68]. The quarkantiquark pair from the virtual gluon combines with spectator quarks and antiquarks from the initial mesons 
to form three final mesons. The combination involves multigluon exchange between the quark and the antiquark, and confinement sets in. While the final mesons are formed, the wave functions are determined.

If two or more mesons are produced in a reaction or a decay, they interact with each other before being detected. The role of final state interactions has been studied in chiral perturbation theory. While two mesons are produced in a photon-photon reaction, final state interactions come from meson loops and meson resonances between the two photons and the final mesons [69,70]. In reproducing experimental data of $\gamma \gamma$ cross sections, final state interactions are essential. However, in the reaction $\pi N \rightarrow \pi \pi N$ final state interactions cause a correction less than $20 \%$ when the total center-of-mass energy of initial $\pi N$ is smaller than $2 \mathrm{GeV}$ [71]. In the hadronic decays $\eta \rightarrow 3 \pi, \eta^{\prime} \rightarrow 3 \pi$, and $\eta^{\prime} \rightarrow \eta \pi \pi$, final state interactions due to loop corrections and resonances lead to excellent agreement of theoretical decay widths with experimental data, $\pi \pi$ rescattering is shown to be important, but $S$-wave $\pi \eta$ rescattering effects are small [72,73]. For decay modes like $D \rightarrow \pi \pi \pi, \quad D \rightarrow \bar{K} \pi \pi, \quad D \rightarrow K K \bar{K}, \quad J / \psi \rightarrow \pi \pi \pi$, $J / \psi \rightarrow \phi \pi \pi, \quad J / \psi \rightarrow \phi K \bar{K}, \quad \bar{B}^{0} \rightarrow \pi \pi \pi, \quad \bar{B}^{0} \rightarrow J / \psi \pi \pi$, $\bar{B}^{0} \rightarrow J / \psi \pi \eta$, and $\bar{B}^{0} \rightarrow J / \psi K \bar{K}$, the decay amplitude is assumed to be linearly dependent on amplitudes of rescattering diagrams of two final mesons since the weak interaction is involved [74-82]. Experimental data on these decays may be accounted for.

In the meson-meson collisions that produce three mesons, final state interactions due to resonances and loop corrections exist. For instance, the $K^{*}$ resonance contributes to $\pi \pi \rightarrow \pi K \bar{K}$ through $\pi \pi \rightarrow K^{*} \bar{K}$ and $K^{*} \rightarrow \pi K$; $\pi K \rightarrow \pi \pi K$ may happen through $\pi K \rightarrow \pi \rho K$ and $\rho K \rightarrow \pi K$. If the final state interactions are taken into account, more accurate cross sections are expected, but we do not include the final state interactions in the present work. When the two initial mesons approach each other, they undergo elastic scattering. The initial state interaction may influence the production of the three final mesons, but we do not include the initial state interaction in the present work.

\section{SUMMARY}

We have proposed a model to study 2-to-3 meson-meson scattering. A gluon is created from a quark or an antiquark constituent, and subsequently the gluon splits into a quark and an antiquark. This process causes a meson-meson collision to produce three mesons. The transition potential for the process has been derived from the Feynman rules in perturbative QCD. Eight Feynman diagrams at tree level are involved in the 2-to-3 meson-meson scattering. From the $S$-matrix element we have derived the transition amplitudes corresponding to the eight Feynman diagrams, and from the eight transition amplitudes we have derived the unpolarized cross section. The 2-to-3 reactions among pions and kaons include $\pi \pi \rightarrow \pi K \bar{K}, \pi K \rightarrow \pi \pi K$, $\pi K \rightarrow K K \bar{K}, K K \rightarrow \pi K K$, and $K \bar{K} \rightarrow \pi K \bar{K}$. We have calculated color, spin, and flavor matrix elements for these reactions. From the cross-section formulas we have obtained numerical unpolarized cross sections for eight isospin channels of the reactions, and the numerical cross section results are parametrized. At zero temperature our cross sections for $\pi K \rightarrow \pi \pi K$ for $I=3 / 2$ are near the experimental data. The unpolarized cross sections depend on temperature. The cross section for any isospin channel at a given temperature has a maximum, and the peak cross section of any reaction decreases as the temperature approaches the critical temperature. By comparison with inelastic 2-to-2 meson-meson scattering, we find that 2-to-3 meson-meson scattering may be as important as inelastic 2-to-2 meson-meson scattering.

\section{ACKNOWLEDGMENTS}

This work was supported by the National Natural Science Foundation of China under Grant No. 11175111.
[1] J. Alitti, J. P. Baton, A. Berthelot, B. Deler, W. J. Fickinger, N. Neveu-René, V. Alles-Borelli, R. Gessaroli, A. Romano, and P. Waloschek, Nuovo Cimento 35, 1 (1965).

[2] E. Colton, E. Malamud, P. E. Schlein, A. D. Johnson, V. J. Stenger, and P. G. Wohlmut, Phys. Rev. D 3, 2028 (1971).

[3] M. J. Losty, V. Chaloupka, A. Ferrando, L. Montanet, E. Paul, D. Yaffe, A. Zieminski, J. Alitti, B. Gandois, and J. Louie, Nucl. Phys. B69, 185 (1974).

[4] N. M. Cason, P. E. Cannata, A. E. Baumbaugh, J. M. Bishop, N. N. Biswas, L. J. Dauwe, V. P. Kenney, R. C.
Ruchti, W. D. Shephard, and J. M. Watson, Phys. Rev. D 28, 1586 (1983).

[5] Aachen-Berlin-Birmingham-Bonn-Hamburg-LondonMünchen Collaboration, Phys. Rev. 138, B897 (1965).

[6] W. Hoogland et al., Nucl. Phys. B126, 109 (1977).

[7] E. A. Alekseeva, A. A. Kartamyshev, V. K. Makar'in, K. N. Mukhin, O. O. Patarakin, M. M. Sulkovskaya, A. F. Sustavov, L. V. Surkova, and L. A. Chernysheva, Sov. Phys. JETP 55, 591 (1982).

[8] K. Takamatsu, Nucl. Phys. A675, 312c (2000).

[9] D. Cohen, T. Ferbel, P. Slattery, and B. Werner, Phys. Rev. D 7, 661 (1973). 
[10] N. B. Durusoy, M. Baubillier, R. George, M. Goldberg, A. M. Touchard, N. Armenise, M. T. Fogli-Muciaccia, and A. Silvestri, Phys. Lett. B 45, 517 (1973).

[11] S. D. Protopopescu, M. Alston-Garnjost, A. BarbaroGaltieri, S. M. Flatté, J. H. Friedman, T. A. Lasinski, G. R. Lynch, M. S. Rabin, and F. T. Solmitz, Phys. Rev. D 7, 1279 (1973).

[12] J. P. Baton, G. Laurens, and J. Reignier, Nucl. Phys. B3, 349 (1967); Phys. Lett. 33B, 528 (1970).

[13] W. D. Walker, J. Carroll, A. Garfinkel, and B. Y. Oh, Phys. Rev. Lett. 18, 630 (1967).

[14] B. Y. Oh, A. F. Garfinkel, R. Morse, W. D. Walker, J. D. Prentice, E. C. West, and T. S. Yoon, Phys. Rev. D 1, 2494 (1970).

[15] B. Hyams et al., Nucl. Phys. B64, 134 (1973).

[16] G. Grayer et al., Nucl. Phys. B75, 189 (1974).

[17] P. Estabrooks and A. D. Martin, Nucl. Phys. B79, 301 (1974).

[18] V. Srinivasan et al., Phys. Rev. D 12, 681 (1975).

[19] C. D. Froggatt and J. L. Petersen, Nucl. Phys. B129, 89 (1977).

[20] A. A. Bel'kov, S. A. Bunyatov, K. N. Mukhin, O. O. Patarakin, V. M. Sidorov, M. M. Sulkovskaya, A. F. Sustavov, and V. A. Yarba, JETP Lett. 29, 597 (1979).

[21] R. Kamiński, L. Leśniak, and K. Rybicki, Z. Phys. C 74, 79 (1997).

[22] L. Rosselet et al., Phys. Rev. D 15, 574 (1977).

[23] S. Pislak et al., Phys. Rev. D 67, 072004 (2003).

[24] R. García-Martín, R. Kamiński, J. R. Peláez, J. R. de Elvira, and F. J. Ynduráin, Phys. Rev. D 83, 074004 (2011).

[25] J. R. Batley et al., Eur. Phys. J. C 54, 411 (2008).

[26] R. Mercer et al., Nucl. Phys. B32, 381 (1971).

[27] B. Jongejans, R. A. van Meurs, A. G. Tenner, H. Voorthuis, P. M. Heinen, W. J. Metzger, H. G. J. M. Tiecke, and R. T. Van de Walle, Nucl. Phys. B67, 381 (1973).

[28] D. Linglin et al., Nucl. Phys. B57, 64 (1973).

[29] P. Estabrooks, R. K. Carnegie, A. D. Martin, W. M. Dunwoodie, T. A. Lasinski, and D. W. G. S. Leith, Nucl. Phys. B133, 490 (1978).

[30] D. Aston et al., Nucl. Phys. B296, 493 (1988).

[31] P. del Amo Sanchez et al., Phys. Rev. D 83, 072001 (2011).

[32] D. Cohen, D. S. Ayres, R. Diebold, S. L. Kramer, A. J. Pawlicki, and A. B. Wicklund, Phys. Rev. D 22, 2595 (1980).

[33] S. M. Roy, Phys. Lett. B 36, 353 (1971); M. R. Pennington and S. D. Protopopescu, Phys. Rev. D 7, 1429 (1973); B. Ananthanarayan, Phys. Rev. D 58, 036002 (1998); G. Colangelo, J. Gasser, and H. Leutwyler, Nucl. Phys. B603, 125 (2001); R. Kamiński, L. Leśniak, and B. Loiseau, Phys. Lett. B 551, 241 (2003).

[34] J. Gasser and H. Leutwyler, Ann. Phys. (N.Y.) 158, 142 (1984); Nucl. Phys. B250, 465 (1985); J. Gasser and U. G. Meissner, Phys. Lett. B 258, 219 (1991); Nucl. Phys. B357, 90 (1991); I. Bijnens, G. Colangelo, G. Ecker, J. Gasser, and M. E. Sainio, Nucl. Phys. B508, 263 (1997).

[35] T. N. Truong, Phys. Rev. Lett. 61, 2526 (1988); A. Dobado, M. J. Herrero, and T. N. Truong, Phys. Lett. B 235, 134 (1990); S. Willenbrock, Phys. Rev. D 43, 1710 (1991); G.-Y. Qin, W. Z. Deng, Z. G. Xiao, and H. Q. Zheng, Phys. Lett. B 542, 89 (2002).
[36] T. N. Truong, Phys. Rev. Lett. 67, 2260 (1991); T. Hannah, Phys. Rev. D 55, 5613 (1997); A. Dobado and J. R. Peláez, Phys. Rev. D 56, 3057 (1997); M. Boglione and M. R. Pennington, Z. Phys. C 75, 113 (1997); J. A. Oller, E. Oset, and J. R. Peláez, Phys. Rev. D 59, 074001 (1999); J. Nieves, M. P. Valderrama, and E. R. Arriola, Phys. Rev. D 65, 036002 (2002); J. Nebreda and J. R. Peláez, Phys. Rev. D 81, 054035 (2010); M. Döring and U.-G. Meißner, J. High Energy Phys. 01 (2012) 009.

[37] F. Guerrero and J. A. Oller, Nucl. Phys. B537, 459 (1999); A. G. Nicola and J. Peláez, Phys. Rev. D 65, 054009 (2002).

[38] B. S. Zou and D. V. Bugg, Phys. Rev. D 50, 591 (1994); L. Li, B. S. Zou, and G.-L. Li, Phys. Rev. D 67, 034025 (2003); F. Q. Wu, B. S. Zou, L. Li, and D. V. Bugg, Nucl. Phys. A735, 111 (2004).

[39] A. Dobado and J. R. Peláez, Phys. Lett. B 286, 136 (1992); A. Dobado and J. Morales, Phys. Rev. D 52, 2878 (1995).

[40] G. Janssen, B. C. Pearce, K. Holinde, and J. Speth, Phys. Rev. D 52, 2690 (1995); B. A. Li, Phys. Rev. D 52, 5165 (1995); B. A. Li, D.-N. Gao, and M.-L. Yan, Phys. Rev. D 58, 094031 (1998); D. Black, A. H. Fariborz, and J. Schechter, Phys. Rev. D 61, 074030 (2000).

[41] J. A. Oller and E. Oset, Nucl. Phys. A620, 438 (1997); F.-K. Guo, R.-G. Ping, P.-N. Shen, H.-C. Chiang, and B. S. Zou, Nucl. Phys. A773, 78 (2006); I. V. Danilkin, L. I. R. Gil, and M. F. M. Lutz, Phys. Lett. B 703, 504 (2011); Z.-H. Guo, L. Liu, U.-G. Meißner, J. A. Oller, and A. Rusetsky, Phys. Rev. D 95, 054004 (2017); I. V. Danilkin and M. Vanderhaeghen, Phys. Lett. B 789, 366 (2019).

[42] J. S. Borges, J. S. Barbosa, and V. Oguri, Phys. Lett. B 393, 413 (1997); 412, 389 (1997).

[43] J. V. Steele, H. Yamagishi, and I. Zahed, Nucl. Phys. A615, 305 (1997).

[44] J. Nieves and E. R. Arriola, Phys. Lett. B 455, 30 (1999); M. Albaladejo, J. A. Oller, E. Oset, G. Rios, and L. Roca, J. High Energy Phys. 08 (2012) 071.

[45] J. A. Oller and E. Oset, Phys. Rev. D 60, 074023 (1999); M. Albaladejo, J. A. Oller, and L. Roca, Phys. Rev. D 82, 094019 (2010).

[46] T. Barnes and E. S. Swanson, Phys. Rev. D 46, 131 (1992); E. S. Swanson, Ann. Phys. (N.Y.) 220, 73 (1992); T. Barnes, E. S. Swanson, and J. Weinstein, Phys. Rev. D 46, 4868 (1992); T. Barnes, N. Black, and E. S. Swanson, Phys. Rev. C 63, 025204 (2001).

[47] V. Flaminio, W. G. Moorhead, D. R. O. Morrison, and N. Rivoire, CERN Report No. CERN-HERA-84-01, 1984.

[48] C. M. Ko, Phys. Rev. C 23, 2760 (1981).

[49] K. Yang, X.-M. Xu, and H. J. Weber, Phys. Rev. D 96, 114025 (2017).

[50] D. Lohse, J. W. Durso, K. Holinde, and J. Speth, Nucl. Phys. A516, 513 (1990).

[51] G. E. Brown, C. M. Ko, Z. G. Wu, and L. H. Xia, Phys. Rev. C 43, 1881 (1991).

[52] Y.-Q. Li and X.-M. Xu, Nucl. Phys. A794, 210 (2007); Z.-Y. Shen and X.-M. Xu, J. Korean Phys. Soc. 66, 754 (2015).

[53] Z.-Y. Shen, X.-M. Xu, and H. J. Weber, Phys. Rev. D 94, 034030 (2016); T.-T. Wang and X.-M. Xu, Chin. Phys. C 43, 024102 (2019).

[54] J. R. Peláez and A. Rodas, Eur. Phys. J. C 78, 897 (2018). 
[55] M. Albaladejo and J. A. Oller, Phys. Rev. Lett. 101, 252002 (2008).

[56] ATLAS Collaboration, Phys. Lett. B 790, 108 (2019).

[57] CMS Collaboration, J. High Energy Phys. 10 (2018) 138.

[58] ALICE Collaboration, Phys. Lett. B 788, 166 (2019).

[59] J.D. Bjorken and S. D. Drell, Relativistic Quantum Mechanics (McGraw-Hill, New York, 1964).

[60] T. Muta, Foundations of Quantum Chromodynamics (World Scientific, Singapore, 1987).

[61] S.-T. Ji, Z.-Y. Shen, and X.-M. Xu, J. Phys. G 42, 095110 (2015).

[62] F. Karsch, E. Laermann, and A. Peikert, Nucl. Phys. B605, 579 (2001).

[63] W. Buchmüller and S.-H. H. Tye, Phys. Rev. D 24, 132 (1981).

[64] K. Nakamura et al. (Particle Data Group), J. Phys. G 37, 075021 (2010).

[65] P. A. Baikov, K. G. Chetyrkin, and J. H. Kühn, Phys. Rev. Lett. 101, 012002 (2008).

[66] M. Davier, S. Descotes-Genon, A. Höcker, B. Malaescu, and Z. Zhang, Eur. Phys. J. C 56, 305 (2008).

[67] P. Kroll, B. Quadder, and W. Schweiger, Nucl. Phys. B316, 373 (1989).

[68] A. T. Goritschnig, P. Kroll, and W. Schweiger, Eur. Phys. J. A 42, 43 (2009).
[69] J. A. Oller and E. Oset, Nucl. Phys. A629, 739 (1998).

[70] G. Mennessier, S. Narison, and X.-G. Wang, Phys. Lett. B 696, 40 (2011).

[71] I. J. R. Aitchison and J. J. Brehm, Phys. Lett. 84B, 349 (1979).

[72] B. Borasoy and R. Nißler, Eur. Phys. J. A 26, 383 (2005).

[73] S. Gonzàlez-Solis and E. Passemar, Eur. Phys. J. C 78, 758 (2018).

[74] J. A. Oller, Phys. Rev. D 71, 054030 (2005).

[75] S. X. Nakamura, Phys. Rev. D 93, 014005 (2016).

[76] R. T. Aoude, P. C. Magalhães, A. C. dos Reis, and M. R. Robilotta, Phys. Rev. D 98, 056021 (2018).

[77] D. Morgan and M. R. Pennington, Phys. Rev. D 48, 1185 (1993).

[78] U.-G. Meißner and J. A. Oller, Nucl. Phys. A679, 671 (2001).

[79] L. Roca, J. E. Palomar, E. Oset, and H. C. Chiang, Nucl. Phys. A744, 127 (2004).

[80] P. Guo, R. Mitchell, and A. P. Szczepaniak, Phys. Rev. D 82, 094002 (2010).

[81] S. Gardner and U.-G. Meißner, Phys. Rev. D 65, 094004 (2002).

[82] M. Albaladejo, J. T. Daub, C. Hanhart, B. Kubis, and B. Moussallam, J. High Energy Phys. 04 (2017) 010. 